\title{
Non-Standard Gauge-Boson Self-Interactions within a Gauge Invariant Model*
}

\author{
Carsten Grosse-Knetter† \\ Ingolf Kuss ${ }^{\ddagger}$ \\ and \\ Dieter Schildknecht \\ Universität Bielefeld \\ Fakultät für Physik \\ 33501 Bielefeld \\ Germany
}

BI-TP 93/15

hep-ph/9304281

April 1993

(Updated Version)

\begin{abstract}
We examine dimension-six extensions of the standard electroweak Lagrangian which are invariant under local $\mathrm{SU}(2)_{\mathrm{L}} \times \mathrm{U}(1)_{\mathrm{Y}}$-transformations. The dimensionfour trilinear and quadrilinear effective interactions of the vector bosons with one another are found to coincide with the vector boson interactions previously derived from global $\mathrm{SU}(2)$ weak isospin symmetry broken by electromagnetism. Supplementing the model by a well-known dimension-six single-parameter quadrupole interaction leads to the most general vector boson self-couplings that can be obtained by addition of dimension-six terms to the standard Lagrangian. We examine in some detail another $\mathrm{SU}(2)_{\mathrm{L}} \times \mathrm{U}(1)_{\mathrm{Y}}$-symmetric interaction which contains $W_{3} B$ mixing and modifies both vector boson self-couplings and fermionic interactions. Independently of being strongly constrained by the LEP 1 data, the addition of this interaction to the above-mentioned non-standard ones does not change the form of the trilinear and quadrilinear non-standard self-couplings of the vector bosons. Therefore, while being interesting in itself with respect to LEP 1 physics, this term is irrelevant with respect to the phenomenology of the vector-boson self-interactions.
\end{abstract}

\footnotetext{
*Partially supported by Deutsche Forschungsgemeinschaft

${ }^{\dagger}$ E-Mail: knetter@physw.uni-bielefeld.de

${ }^{\ddagger}$ E-Mail: kuss@physf.uni-bielefeld.de
} 


\section{Introduction}

The body of presently available data [1] on electroweak phenomena is in excellent agreement with the predictions of the standard $\mathrm{SU}(2)_{\mathrm{L}} \times \mathrm{U}(1)_{\mathrm{Y}}$ electroweak theory [2]. The agreement between theory and experiment is particularly noteworthy with respect to the LEP 1 data which are precise enough to be sensitive to effects beyond the simple tree approximation of the standard Lagrangian.

Concerning loop effects, it must be stressed, however, that the agreement between theory and experiment is largely based on the well-understood QED contribution of fermion loops to the photon propagator, the "running" of the electromagnetic fine structure constant, i.e., $\alpha\left(M_{Z}^{2}\right)=\frac{1}{128.9}$, and the violation of global $\mathrm{SU}(2)$ symmetry induced by the heavy-topquark-loop. Quantitatively, this is seen by comparing the exact one loop predictions with the ones of the dominant-fermion-loop approximation [3, 4]. One finds that the contribution of the $M_{H}$-dependent bosonic loop corrections is of the order of magnitude of the LEP 1 experimental errors. The small bosonic loop corrections appear as a consequence of the renormalizable non-Abelian $\mathrm{SU}(2)_{\mathrm{L}} \times \mathrm{U}(1)_{\mathrm{Y}}$ structure of the couplings of the vector bosons with one another and the assumed existence of the Higgs scalar particle. The consistency between theory and the LEP 1 precision data may thus be considered to be a major triumph of the standard model, although the bosonic loop corrections have not been quantitatively determined, as evidenced by the uncertainty in the Higgs-boson mass, $M_{H}$, extracted from the LEP 1 precision data.

The fact that the boson-loop vacuum-polarization effects and, accordingly, the trilinear and quadrilinear couplings among the vector bosons are not precisely determined by the LEP 1 data provides sufficient reason to perform direct measurements of the trilinear self-couplings in $e^{+} e^{-} \rightarrow W^{+} W^{-}$[5, 6] and of the quadrilinear ones in $e^{+} e^{-} \rightarrow$ $W^{+} W^{-} Z, W^{+} W^{-} \gamma[7,8]$. Such a program can be started at LEP 2 and continued in the more distant future at a linear $e^{+} e^{-}$collider with an energy of, e.g., $\sqrt{s}=500 \mathrm{GeV}$ (NLC) 9. 10.

On the other hand, even though any theory which correctly describes the interactions of the vector bosons with the leptons and the quarks and which yields sufficiently small deviations from the standard model in the sector of gauge-boson self-interactions would be compatible with the LEP 1 data, it is evidently an important question to be asked, whether at all we can think of alternatives to the standard-model couplings among vector bosons which fulfil the LEP 1 constraints.

Some time ago, it was suggested (KMSS model [11, 12]) that vector-boson self-couplings alternative to the standard ones can indeed be introduced with a certain amount of credibility, provided a minimum amount of symmetry is required for the couplings in addition to Lorentz invariance and electromagnetic gauge invariance. Restricting oneself to dimensionfour terms in the bosonic Lagrangian and requiring that global SU(2) weak isospin symmetry be broken by electromagnetism only (by $\gamma-W^{3}$ current mixing, in particular), one easily reconstructs the standard model in the fermion sector, but obtains an alternative to the standard model for the trilinear couplings of the vector bosons to one another. While Lorentz invariance and electromagnetic gauge invariance in general allow for three independent dimension-four trilinear couplings among the vector bosons, the underlying global $\mathrm{SU}(2)$ weak isospin symmetry in the KMSS model restricts the number of free couplings to two independent ones. Dimension-four trilinear and quadrilinear self-couplings altogether are described in terms of four free parameters. The standard $\mathrm{SU}(2)_{\mathrm{L}} \times \mathrm{U}(1)_{\mathrm{Y}}$ theory is recovered for a specific choice of these four parameters.

In the present work, we will examine a dimension-six extension of the standard mo- 
del, which is invariant under local $\mathrm{SU}(2)_{\mathrm{L}} \times \mathrm{U}(1)_{\mathrm{Y}}$ transformations. It contains two free coupling constants and the Higgs scalar doublet in an essential manner. We will find that the trilinear couplings of the vector bosons to one another, and also the quadrilinear couplings of the abovementioned KMSS model, provided certain qualifications are valid, are precisely reproduced by this dimension-six $\mathrm{SU}(2)_{\mathrm{L}} \times \mathrm{U}(1)_{\mathrm{Y}}$ invariant extension of the standard Lagrangian. Originally [11], no use of the Higgs scalar was made, deliberately, in the ansatz for the KMSS Lagrangian. One may in fact even challenge the very idea of introducing dimension-six nonrenormalizable Higgs interactions, as the Higgs scalar was introduced originally to assure renormalizability. Nevertheless, the introduction of non-standard Higgs interactions in the KMSS model reduces the cut-off-dependent bosonic loop divergences in physical quantities to logarithmic ones and, accordingly, protects its predictions from disagreeing with the LEP 1 empirical data [13, 14]. On the other hand, in $e^{+} e^{-} \rightarrow W^{+} W^{-}$and $e^{+} e^{-} \rightarrow W^{+} W^{-}\left(\gamma, Z^{0}\right)$, the non-standard couplings lead to deviations from the standardmodel predictions which increase with the square of the $e^{+} e^{-}$center-of-mass energy. The KMSS model thus provides an excellent testing ground when empirically exploring the vector-boson self interactions by measuring $e^{+} e^{-} \rightarrow W^{+} W^{-}$, a reaction in which the Higgs interactions are negligible. It will allow one to precisely quantify [6] to what extent future data agree with, e.g., the standard-model predictions.

A more general model with non-standard couplings among the electroweak vector bosons is obtained if the KMSS Lagrangian is extended by adding a dimension-six quadrupole interaction among the vector bosons. It is well known [15] that the appropriate use of non-Abelian field-strength tensors allows one to introduce a quadrupole interaction which is invariant under local $\mathrm{SU}(2)_{\mathrm{L}} \times \mathrm{U}(1)_{\mathrm{Y}}$ transformations and determined by a single free parameter for both the $\gamma W^{+} W^{-}$and the $Z^{0} W^{+} W^{-}$interactions. We will convince ourselves that the resulting three-free-parameter model is indeed the most general $S U(2)_{L} \times U(1)_{Y}$-symmetric dimension-six extension of the standard theory containing non-standard vector-boson selfinteractions, provided the vector-boson-fermion sector is required to remain unmodified and higher derivatives as well as $C$ - and $C P$ - violating terms are excluded.

Another single-parameter dimension-six extension of the standard model, which, however, affects vector-boson fermion interactions apart from the interactions of the vector bosons among each other, has recently received much attention [16]. Essentially it consists of adding a $W^{3} B$ current-mixing term to the standard Lagrangian. The mixing term is accompanied by non-standard Higgs interactions and non-standard trilinear couplings of the vector-bosons to one another in such a manner that the resulting interaction becomes invariant under local $\mathrm{SU}(2)_{\mathrm{L}} \times \mathrm{U}(1)_{\mathrm{Y}}$ transformations. We will demonstrate that the $W^{3} B$ current-mixing term in the Lagrangian can be removed by an appropriate diagonalization procedure. Apart from the (standard and non-standard) interactions of the Higgs scalar particle, the model, upon diagonalisation, turns out to be a revival of the Hung-Sakurai Lagrangian [17]. This model in itself is of much interest, as it allows one to quantify «, 18] deviations from the standard $\mathrm{SU}(2)_{\mathrm{L}} \times \mathrm{U}(1)_{\mathrm{Y}}$ symmetry of vector-boson fermion interactions. For $e^{+} e^{-} \rightarrow W^{+} W^{-}$, the Hung-Sakurai model leads to a fairly decent high-energy behaviour [17], a feature rediscovered in [16] and erraneously uniquely associated with the local $\mathrm{SU}(2)_{\mathrm{L}} \times \mathrm{U}(1)_{\mathrm{Y}}$ symmetry obtained when introducing non-standard Higgs interactions in addition to formulating $W^{3} B$ current mixing with the non-Abelian $\mathrm{W}$-field tensor. The additional Higgs interactions are irrelevant, however, for the behaviour of the cross section for $e^{+} e^{-} \rightarrow W^{+} W^{-}$. Quite apart from the fact that $W^{3} B$ mixing is strongly constrained 44, 16, 18] by the LEP 1 data, it will be shown that the omission of an additional $W^{3} B$ mixing term does not lead to any loss of generality concerning the empirical implications of the aforementioned general three-parameter model with respect to non-standard vector- 
boson self-couplings, since the form of the relations among the non-standard couplings is independent of the presence of $\mathcal{L}_{W B}$.

In section 2, we briefly review the parametrization of the trilinear couplings of the vector bosons among one another. In section 3, we rederive the KMSS model by extending the standard model by interaction terms, which are invariant under local $\mathrm{SU}(2)_{\mathrm{L}} \times \mathrm{U}(1)_{\mathrm{Y}}$ transformations. In section 4 , we add the $\mathrm{SU}(2)_{\mathrm{L}} \times \mathrm{U}(1)_{\mathrm{Y}}$-invariant quadrupole term to the Lagrangian and comment on the generality of the so-defined three-free-parameter Lagrangian. Various additional terms can be disregarded, as they can simply be removed by field and coupling-constant redefinitions. The recent claim [16] that certain non-standard terms are a priori "unnatural" is commented upon. Section 5 is devoted to a thorough discussion of the $W^{3} B$ current-mixing extension of the standard model. Final conclusions will be drawn in section 6 .

\section{Parametrization of Trilinear Vector-Boson Self- Couplings}

If we disregard the theoretically somewhat remote $\mathrm{CP}$-violating couplings ${ }^{1}$ of the vector bosons with one another and a C-violating anapole term, the most general trilinear couplings of electroweak vector bosons are contained in the effective Lagrangian (e.g., [20])

$$
\begin{aligned}
\mathcal{L}_{3 G B}= & -i e\left[A_{\mu}\left(W^{-\mu \nu} W_{\nu}^{+}-W^{+\mu \nu} W_{\nu}^{-}\right)+\kappa_{\gamma} A_{\mu \nu} W^{+\mu} W^{-\nu}\right] \\
& -i e g_{Z W W}\left[Z_{\mu}\left(W^{-\mu \nu} W_{\nu}^{+}-W^{+\mu \nu} W_{\nu}^{-}\right)+\kappa_{Z} Z_{\mu \nu} W^{+\mu} W^{-\nu}\right] \\
& +i e \frac{\lambda_{\gamma}}{M_{W}^{2}} A_{\mu}{ }^{\nu} W_{\nu}^{-\lambda} W_{\lambda}^{+\mu} \\
& +i e \frac{\cos \theta_{W}}{\sin \theta_{W}} \frac{\lambda_{Z}}{M_{W}^{2}} Z_{\mu}{ }^{\nu} W_{\nu}^{-\lambda} W_{\lambda}^{+\mu}
\end{aligned}
$$

where $W_{\mu \nu}^{+}=\partial_{\mu} W_{\nu}^{+}-\partial_{\nu} W_{\mu}^{+}$, etc., and $g_{Z W W}, \kappa_{\gamma}$ and $\kappa_{Z}$ denote the $Z W^{+} W^{-}$coupling and the electromagnetic and weak dipole moment, respectively. The strengths of the dimensionsix quadrupole terms, not present in the standard model, are denoted by $\lambda_{\gamma}$ and $\lambda_{Z}$. It is convenient to rewrite (2.1) in a form [6] which is linear in the deviations from the standard couplings (given by $g_{Z W W}=\frac{\sin \theta_{W}}{\cos \theta_{W}}, \kappa_{\gamma}=\kappa_{Z}=1$ and $\lambda_{\gamma}=\lambda_{Z}=0$ ), i.e.,

$$
\begin{aligned}
\mathcal{L}_{3 G B}= & -i e\left[A_{\mu}\left(W^{-\mu \nu} W_{\nu}^{+}-W^{+\mu \nu} W_{\nu}^{-}\right)+A_{\mu \nu} W^{+\mu} W^{-\nu}\right] \\
& -i e x_{\gamma} A_{\mu \nu} W^{+\mu} W^{-\nu} \\
& -i e\left(\frac{\cos \theta_{W}}{\sin \theta_{W}}+\delta_{Z}\right)\left[Z_{\mu}\left(W^{-\mu \nu} W_{\nu}^{+}-W^{+\mu \nu} W_{\nu}^{-}\right)+Z_{\mu \nu} W^{+\mu} W^{-\nu}\right] \\
& -i e x_{Z} Z_{\mu \nu} W^{+\mu} W^{-\nu} \\
& +i e \frac{y_{\gamma}}{M_{W}^{2}} A_{\mu}{ }^{\nu} W_{\nu}^{-\lambda} W_{\lambda}^{+\mu} \\
& +i e \frac{y_{Z}}{M_{W}^{2}} Z_{\mu}{ }^{\nu} W_{\nu}^{-\lambda} W_{\lambda}^{+\mu}
\end{aligned}
$$

where

$$
\delta_{Z}=g_{Z W W}-\frac{\cos \theta_{W}}{\sin \theta_{W}}
$$

\footnotetext{
${ }^{1}$ Compare, however, ref. [19 for an unambiguous empirical test of $C P$ invariance via comparison of certain $W^{+}$and $W^{-}$density-matrix elements.
} 


$$
\begin{aligned}
x_{\gamma} & =\kappa_{\gamma}-1, \\
x_{Z} & =g_{Z W W}\left(\kappa_{Z}-1\right), \\
y_{\gamma} & =\lambda_{\gamma} \\
y_{Z} & =\frac{\cos \theta_{W}}{\sin \theta_{W}} \lambda_{Z} .
\end{aligned}
$$

In (2.1) to (2.3) $\sin \theta_{W} \equiv \frac{e}{g}$, where $e$ is the electromagnetic coupling strength and $g$ denotes the coupling of the $W$-field to the weak isospin current. Non-standard trilinear couplings will be expressed in the form (2.2) in the subsequent sections.

\section{The KMSS Model}

Several years ago, a model of trilinear and quadrilinear vector-boson self-interactions was suggested [11] which contains four free parameters, two of these in the trilinear sector. The model is based on the minimal set of assumptions necessary for an empirically succesful description of the vector-boson-fermion interactions. It extrapolates into the bosonic sector of the theory. In the bosonic sector all dimension-four interactions among vector bosons which fulfil a global SU(2) weak-isospin symmetry are allowed. This symmetry is broken by the (primordial) photon, in particular via mixing in the neutral sector. For an appropriate choice of the mixing strength, one recovers the standard vector-boson-fermion interactions, but obtains non-standard interactions of the vector bosons with one another. The latter ones are given by 11

$$
\begin{aligned}
\mathcal{L}_{K M S S}= & -i e A_{\mu}\left(W^{-\mu \nu} W_{\nu}^{+}-W^{+\mu \nu} W_{\nu}^{-}\right)-i e \kappa_{\gamma} A_{\mu \nu} W^{+\mu} W^{-\nu} \\
& +i\left(e \frac{\sin \theta_{W}}{\cos \theta_{W}}-\frac{\hat{g}}{\cos \theta_{W}}\right) Z_{\mu}\left(W^{-\mu \nu} W_{\nu}^{+}-W^{+\mu \nu} W_{\nu}^{-}\right) \\
& +i\left(e \kappa_{\gamma} \frac{\sin \theta_{W}}{\cos \theta_{W}}-\frac{\hat{g}}{\cos \theta_{W}}\right) Z_{\mu \nu} W^{+\mu} W^{-\nu} \\
& -e^{2}\left(A_{\mu} A^{\mu} W_{\nu}^{+} W^{-\nu}-A_{\mu} A_{\nu} W^{+\mu} W^{-\nu}\right) \\
& +2 e\left(e \frac{\sin \theta_{W}}{\cos \theta_{W}}-\frac{\hat{g}}{\cos \theta_{W}}\right)\left(A_{\mu} Z^{\mu} W_{\nu}^{+} W^{-\nu}-\frac{1}{2} A_{\mu} Z_{\nu}\left(W^{+\mu} W^{-\nu}+W^{-\mu} W^{+\nu}\right)\right) \\
& -\left[e\left(e \frac{\sin ^{2} \theta_{W}}{\cos \theta_{W}}-2 \hat{g} \frac{\sin \theta_{W}}{\cos ^{2} \theta_{W}}\right)+\frac{\hat{\hat{g}}}{\cos ^{2} \theta_{W}}\right]\left(Z_{\mu} Z^{\mu} W_{\nu}^{+} W^{-\nu}-Z_{\mu} Z_{\nu} W^{+\mu} W^{-\nu}\right) \\
& -\frac{\tilde{g}}{\cos ^{2} \theta_{W}} Z_{\mu} Z^{\mu} W_{\nu}^{+} W^{-\nu}-\frac{\tilde{g}}{4 \cos ^{2} \theta_{W}} Z_{\mu} Z^{\mu} Z_{\nu} Z^{\nu} \\
& +\frac{1}{2} \hat{\hat{g}}\left(W_{\mu}^{+} W_{\nu}^{-} W^{+\mu} W^{-\nu}-W_{\mu}^{+} W_{\nu}^{-} W^{-\mu} W^{+\nu}\right)-\tilde{g} W_{\mu}^{+} W_{\nu}^{-} W^{-\mu} W^{+\nu} .
\end{aligned}
$$

This Lagrangian contains an arbitrary coupling constant, $\hat{g}$, which characterizes the strength of the SU(2)-invariant trilinear vector boson self-interactions as well as an arbitrary magnetic dipole moment of the $W^{ \pm}$boson, $\kappa_{\gamma}$, and two parameters, $\hat{\hat{g}}$ and $\tilde{g}$, which appear in the quadrilinear interaction terms only. The trilinear part of (3.1) is easily seen to have the form (2.2) with

$$
\begin{aligned}
\delta_{Z} & =\frac{\sin \theta_{W} \frac{\hat{g}}{e}-1}{\cos \theta_{W} \sin \theta_{W}}, \\
x_{\gamma} & =\kappa_{\gamma}-1
\end{aligned}
$$


while $x_{Z}$ is related to $x_{\gamma}$ via

$$
x_{Z}=-x_{\gamma} \frac{\sin \theta_{W}}{\cos \theta_{W}}
$$

The quadrupole interactions are absent in (3.1), i.e. $y_{\gamma}=y_{Z}=0$. Relation (3.3) is a consequence of requiring global $\mathrm{SU}(2)$ symmetry in the limit of vanishing coupling of the $W^{ \pm}$to the (primordial, unmixed) photon. Relation (3.3) can only be violated at the expense of allowing for an extra isolated term of the form

$$
W_{\mu \nu}^{3} W^{+\mu} W^{-\nu}
$$

which violates $\mathrm{SU}(2)$ symmetry intrinsically, i.e., independently of the presence of the (primordial) photon field in the neutral sector. To summarize, the trilinear couplings in the KMSS model are characterized by two free parameters, $\delta_{Z}$ and $x_{\gamma}$, and the relation (3.3) expressing $x_{Z}$ in terms of $x_{\gamma}$.

We turn to a reconstruction of the KMSS Lagrangian (3.1) from a gauge-invariant extension of the standard model. In particular, we consider the Lagrangian

$$
\mathcal{L}_{e f f}=\mathcal{L}_{S M}+\epsilon_{W \Phi} \frac{g}{M_{W}^{2}} \mathcal{L}_{W \Phi}+\epsilon_{B \Phi} \frac{g^{\prime}}{M_{W}^{2}} \mathcal{L}_{B \Phi}
$$

containing the two $S U(2)_{L} \times U(1)_{Y}$-symmetric dimension-six terms

$$
\mathcal{L}_{W \Phi}=i \operatorname{tr}\left[\left(D_{\mu} \Phi\right)^{\dagger} W^{\mu \nu}\left(D_{\nu} \Phi\right)\right]
$$

and

$$
\mathcal{L}_{B \Phi}=-\frac{1}{2} i \operatorname{tr}\left[\tau_{3}\left(D_{\mu} \Phi\right)^{\dagger}\left(D_{\nu} \Phi\right)\right] B^{\mu \nu}
$$

Here, $\Phi$ denotes the standard complex scalar Higgs doublet field,

$$
\Phi=\frac{1}{\sqrt{2}}\left((v+H) \mathbf{1}+i \varphi_{i} \tau_{i}\right)
$$

Its covariant derivative is given by

$$
D_{\mu} \Phi=\partial_{\mu} \Phi+i g W_{\mu} \Phi-\frac{i}{2} g^{\prime} \Phi \tau_{3} B_{\mu}
$$

where

$$
W_{\mu}=\frac{1}{2} W_{\mu i} \tau_{i}
$$

denotes the non-Abelian vector field, and

$$
\begin{aligned}
W_{\mu \nu} & =\partial_{\mu} W_{\nu}-\partial_{\nu} W_{\mu}+i g\left[W_{\mu}, W_{\nu}\right] \\
B_{\mu \nu} & =\partial_{\mu} B_{\nu}-\partial_{\nu} B_{\mu} \nu
\end{aligned}
$$

are the field strength tensors. As usual, $g^{\prime}$ denotes the $\mathrm{U}(1)_{\mathrm{Y}}$ coupling, $g^{\prime}=\frac{e}{\cos \theta_{W}}$. Passing to the physical photon and $Z^{0}$ fields in (3.5), one finds that indeed the trilinear boson sector has the form (2.2) with

$$
\begin{aligned}
\delta_{Z} & =\frac{\epsilon_{W \Phi}}{\sin \theta_{W} \cos \theta_{W}}, \\
x_{\gamma} & =\epsilon_{W \Phi}+\epsilon_{B \Phi},
\end{aligned}
$$

\footnotetext{
${ }^{2}$ Both interactions, $(3.6)$ and $(3.7)$, are contained in the systematic classification of non-standard interaction terms given in 21 .
} 
and the constraint

$$
x_{Z}=-x_{\gamma} \frac{\sin \theta_{W}}{\cos \theta_{W}}
$$

which is identical with (3.3). The trilinear vector-boson interactions described by the $\mathrm{SU}(2)_{\mathrm{L}} \times \mathrm{U}(1)_{\mathrm{Y}}$-invariant Lagrangian (3.5) indeed coincide with the trilinear vector-boson interactions of the KMSS model, (3.1), based on global SU(2) symmetry broken by electromagnetism. This result is of no surprise, as the dimension-six interactions (3.6) and (3.7) upon spontaneous symmetry breaking fulfil the requirement of restoration of global $\mathrm{SU}(2)$ symmetry for vanishing hypercharge coupling constant, $g^{\prime}$. Indeed, the contribution of $\mathcal{L}_{B \phi}$ to the Lagrangian (3.7) vanishes in this limit, while the trilinear couplings contained in $\mathcal{L}_{W \phi}$ have the form

$$
\vec{W}^{\mu \nu}\left(\vec{W}_{\mu} \times \vec{W}_{\nu}\right),
$$

i.e., there is no isolated term of form (3.4) present, which would violate $\mathrm{SU}(2)$ symmetry intrinsically"

The quadrilinear couplings contained in the Lagrangian (3.5) (apart from terms of order $\delta_{Z}^{2}$ ) coincide with the special case of the KMSS model obtained by imposing the conditions

$$
\begin{aligned}
& \hat{\hat{g}}=\hat{g}^{2} \\
& \tilde{g}=0
\end{aligned}
$$

which reduce the number of four free parameters to two independent ones, $\delta_{Z}$ and $x_{\gamma}$.

Using the connection (3.12) between the parameters $\delta_{Z}, x_{\gamma}$ and $\epsilon_{W \phi}, \epsilon_{B \phi}$, the Lagrangian (3.5) may be rewritten in a form which directly allows one to read off its properties with respect to the trilinear boson couplings,

$$
\mathcal{L}=\mathcal{L}_{S M}+\frac{g}{M_{W}^{2}}\left(\delta_{Z} \sin \theta_{W} \cos \theta_{W} \mathcal{L}_{W \phi}+\left(x_{\gamma} \frac{\sin \theta_{W}}{\cos \theta_{W}}-\delta_{Z} \sin ^{2} \theta_{W}\right) \mathcal{L}_{B \phi}\right)
$$

Two special cases are worth noting. The omission of the interaction of the hypercharge field [7], $\mathcal{L}_{B \phi}$, corresponds to

$$
x_{\gamma}=\delta_{Z} \sin \theta_{W} \cos \theta_{W}
$$

and, upon using (3.13),

$$
x_{Z}=-\delta_{Z} \sin ^{2} \theta_{W}
$$

and reduces the model to a single-parameter $\left(\delta_{Z}\right)$ model for trilinear and quadrilinear interactions among the vector bosons. In this case, trilinear and quadrilinear interactions of the vector bosons with one another are such that [22] the most strongly unitarity-violating terms of order $s^{2}$ in tree amplitudes for the scattering of massive vector bosons are absent. Equivalently, quartic divergences in one-loop corrections to the $\rho$-parameter [23] (without any Higgs contribution) are absent if (3.17) is imposed. It was originally this requirement of a (moderately) decent high-energy and loop behaviour by which the condition (3.17) was introduced (BKS model, 22, 23]) in the KMSS model.

A second special case of (3.16) amounts to the omission of $\mathcal{L}_{W \phi}$ via

$$
\delta_{Z}=0
$$

In this case $x_{\gamma}$ only and $x_{Z}=-x_{\gamma}\left(\sin \theta_{W} / \cos \theta_{W}\right)$ remain as non-standard couplings.

\footnotetext{
${ }^{3}$ Note that a term of the form (3.4) can only be obtained by introducing an additional dimension-eight interaction into the Lagrangian, which is given by $i \operatorname{tr}\left[\tau_{3}\left(D_{\mu} \Phi\right)^{\dagger}\left(D_{\nu} \Phi\right)\right] \operatorname{tr}\left[\Phi^{\dagger} W_{\mu \nu} \Phi \tau_{3}\right][27$. This term indeed yields an extra term of the form (3.4), which violates $\mathrm{SU}(2)$ symmetry intrinsically.
} 
With respect to the quadrilinear coupling strengths we noted that the $\mathrm{SU}(2)_{\mathrm{L}} \times \mathrm{U}(1)_{\mathrm{Y}^{-}}$ symmetric Lagrangian (3.5) yields a two-parameter reduction of the quadrilinear couplings in (3.1) which is characterized by the constraints (3.15). For completeness, we remark that the more general four-parameter Lagrangian (3.1) for the couplings of the vector bosons among each other can also be obtained within an $\mathrm{SU}(2)_{\mathrm{L}} \times \mathrm{U}(1)_{\mathrm{Y}}$ symmetric ansatz, at the expense, however, of allowing for dimension-eight terms. One has to add the terms

$$
\begin{aligned}
& \frac{\epsilon_{D \Phi, 1}}{M_{W}^{4}} \mathcal{L}_{D \Phi, 1}=-\frac{\epsilon_{D \Phi, 1}}{4 M_{W}^{4}} \operatorname{tr}\left(D_{\mu} \Phi^{\dagger} D_{\nu} \Phi\right) \operatorname{tr}\left(D^{\mu} \Phi^{\dagger} D^{\nu} \Phi\right) \\
& \frac{\epsilon_{D \Phi, 2}}{M_{W}^{4}} \mathcal{L}_{D \Phi, 2}=-\frac{\epsilon_{D \Phi, 2}}{4 M_{W}^{4}} \operatorname{tr}\left(D_{\mu} \Phi^{\dagger} D^{\mu} \Phi\right) \operatorname{tr}\left(D_{\nu} \Phi^{\dagger} D^{\nu} \Phi\right)
\end{aligned}
$$

to the Lagrangian (3.5) with the identification

$$
\begin{aligned}
\epsilon_{D \Phi, 1} & =2 \hat{g}^{2}-\hat{\hat{g}} \\
\epsilon_{D \Phi, 2} & =\tilde{g}-2 \hat{g}^{2}+\hat{\hat{g}} .
\end{aligned}
$$

In summary, the trilinear and (special values of) the quadrilinear couplings of the vector bosons of the KMSS model are recovered from a Lagrangian which is invariant under local $\mathrm{SU}(2)_{\mathrm{L}} \times \mathrm{U}(1)_{\mathrm{Y}}$ transformations at the expense of introducing dimension-six terms containing non-standard interactions involving the Higgs particle. The interactions of the Higgs scalar are obviously irrelevant for the predictions of the KMSS model for $W^{+} W^{-}$ production in $e^{+} e^{-}$annihilation at LEP2- and higher energies. They are of relevance, however, as far as vector boson loops within the KMSS model are concerned. A recent analysis [13, 14] of loop contributions indeed has explicitly verified that only (mild) logarithmic dependences on the (necessary) cutoff $\Lambda$ remain at the one-loop levelf, apart from a quadratic dependence on the Higgs-boson mass, $M_{H}^{2}$. By supplementing the non-standard two-free-parameter vector-boson interactions of the KMSS model with appropriately chosen non-standard Higgs couplings, one indeed protects the one-loop predictions of the KMSS model from disagreeing with the LEP1 precision data (provided realistic choices of the Higgs mass, $M_{H} \leq 1 \mathrm{TeV}$, are adopted).

In the dimension-six extension of the KMSS model we restricted ourselves to the standard linear realization of the $\mathrm{SU}(2)_{\mathrm{L}} \times \mathrm{U}(1)_{\mathrm{Y}}$ symmetry, spontaneously broken via the Higgs mechanism. It seems appropriate to add a remark on the alternative of realizing the $\mathrm{SU}(2)_{\mathrm{L}} \times$ $\mathrm{U}(1)_{\mathrm{Y}}$ symmetry in a non-linear way [25, 26, 28]. Such a non-linear representation is obtained by replacing the Higgs doublet $\Phi$ in (3.6) and (3.7) by an expression which is nonpolynomial in the pseudo-Goldstone fields, via

$$
\Phi \equiv \frac{1}{\sqrt{2}}\left((v+H) \mathbf{1}+i \varphi_{i} \tau_{i}\right) \rightarrow U \equiv \frac{v}{\sqrt{2}} \exp \left(\frac{i \varphi_{i} \tau_{i}}{v}\right)
$$

The model obtained via this substitution in (3.6) and (3.7) is symmetric under local SU(2) $)_{\mathrm{L}} \times$ $\mathrm{U}(1)_{\mathrm{Y}}$ transformations without containing a physical Higgs particle, and the KMSS model is now obtained by choosing the unitary gauge. Due to the absense of the Higgs scalar, this formulation of the model leads to quadratic divergences at the one-loop level in LEP I observables. The aforementioned quadratic dependence [13, 14] of the loop corrections on the Higgs boson mass, $M_{H}^{2}$, is converted into a $\Lambda^{2}$ divergence, since the nonlinear model is the limit of the linear model for $M_{H} \rightarrow \infty$.

\footnotetext{
${ }^{4}$ According to a most recent analysis in ref. [24], obtained after completion of the present work, this point can even be strengthened. In [24] it is shown that the logarithmic divergences can indeed be absorbed in the renormalization of (standard and non-standard) couplings in the Lagrangian. Even though the relevant non-standard couplings are strongly restricted by the LEP 1 results, practically no conclusion can be drawn on the vector-boson self-couplings, unless one imposes additional assumptions about certain bare couplings.
} 


\section{The General Three-Parameter Model}

\subsection{The Three-Parameter $\mathrm{SU}(2)_{\mathrm{L}} \times \mathrm{U}(1)_{\mathrm{Y}}$-Symmetric Model}

Supplementing the Lagrangian (3.5) by a well known quadrupole term [15] constructed from the non-Abelian field-strength-tensor $W_{\mu \nu}$ (3.11),

$$
\mathcal{L}_{W}=-\frac{2}{3} i \operatorname{tr}\left(W_{\mu}^{\nu} W_{\nu}^{\lambda} W_{\lambda}^{\mu}\right),
$$

we obtain

$$
\mathcal{L}_{\text {eff }}=\mathcal{L}_{S M}+\epsilon_{W \Phi} \frac{g}{M_{W}^{2}} \mathcal{L}_{W \Phi}+\epsilon_{B \Phi} \frac{g^{\prime}}{M_{W}^{2}} \mathcal{L}_{B \Phi}+\epsilon_{W} \frac{g}{M_{W}^{2}} \mathcal{L}_{W}
$$

It turns out that $\epsilon_{W}$ in (4.2) is identical to $y_{\gamma}$ in (2.2),

$$
y_{\gamma}=\epsilon_{W}
$$

while [15]

$$
y_{Z}=y_{\gamma} \frac{\cos \theta_{W}}{\sin \theta_{W}} .
$$

We note that the loop effects on LEP 1 observables due to the quadrupole interaction (4.1) were recently examined in [16. As expected from the underlying local $\mathrm{SU}(2)_{\mathrm{L}} \times \mathrm{U}(1)_{\mathrm{Y}}$ symmetry, an only mild logarithmic cut-off dependence remains after renormalization. In this sense all three non-standard interactions in (4.2) are equivalent to each other, even though, on the other hand, $\mathcal{L}_{W \Phi}$ and $\mathcal{L}_{B \Phi}$ lead to a quadratic dependence of LEP 1observables on $M_{H}^{2}$, while (obviously) no $M_{H}$-dependence is present in the loop corrections from $\mathcal{L}_{W}$.

We will convince ourselves that the addition of the quadrupole interaction to the KMSS model yields the most generalf $\mathrm{SU}(2)_{\mathrm{L}} \times \mathrm{U}(1)_{\mathrm{Y}}$-symmetric dimension-six extension of the standard model as far as the interactions of the vector bosons with one another are concerned, provided a few restrictive assumptions are introduced to be specified immediately. When claiming the generality of (4.2), we are restricting ourselves to adding C- and CPconserving interactions only. Interactions containing higher derivatives are omitted, and we have obviously restricted ourselves to deviations from the standard model which leave the empirically well-known fermion interactions untouched.

The omission of a few particular terms in (4.2), nevertheless, needs to be discussed when claiming generality of (4.2). First of all, there are two dimension-six terms

$$
\begin{aligned}
\mathcal{L}_{W W, 1} & =-\frac{1}{4} \operatorname{tr}\left(\Phi^{\dagger} \Phi\right) \operatorname{tr}\left(W_{\mu \nu} W^{\mu \nu}\right), \\
\mathcal{L}_{W W, 2} & =-\frac{1}{2} \operatorname{tr}\left(\Phi^{\dagger} W_{\mu \nu} W^{\mu \nu} \Phi\right),
\end{aligned}
$$

which contain the non-Abelian field-strength tensor $W_{\mu \nu}$. By carrying out a suitable redefinition of the $W$ field and of the coupling $g$, the terms (4.5) and (4.6) can be shown to be reduced to non-standard interactions containing the Higgs scalar, while neither the fermionic

\footnotetext{
${ }^{5}$ Any effective Lagrangian with arbitrary self interactions can be embedded into an $\mathrm{SU}(2)_{\mathrm{L}} \times \mathrm{U}(1)_{\mathrm{Y}}$ symmetric framework [27, 26, 28] provided terms of dimension higher than six are allowed. The restriction to dimension-six terms is motivated by simplicity. Moreover, terms of higher dimension, despite an underlying gauge symmetry, presumably imply more seriously divergent loop contributions, or rather higher powers of the Higgs mass in observables, and are theoretically and phenomenologically disfavoured.
} 
interactions nor the interactions of the vector bosons among each other are affected. The terms (4.5) and (4.6) can be neglected without loss of generality.

Another interesting dimension-six interaction is given by

$$
\mathcal{L}_{W B}=-\frac{1}{4} \operatorname{tr}\left(\Phi^{\dagger} W_{\mu \nu} \Phi \tau_{3}\right) B^{\mu \nu}
$$

It contains $W^{3} B$ mixing and in addition a non-standard $B W^{+} W^{-}$interaction (apart from non-standard Higgs terms). As mentioned in the introduction, this interaction, when added to the standard Lagrangian, yields the Hung-Sakurai electroweak model [17] supplemented by non-standard Higgs interactions. The term (4.7) is of interest with respect to LEP 1 physics (in fact, its strength is strongly suppressed [ [1, 18] from LEP 1 data), but irrelevant with respect to non-standard boson couplings, as its addition to (4.2) leaves the form of the non-standard vector-boson self-interactions unchanged. This will be discussed thoroughly in section 5 .

With respect to the point of view recently expressed, that the interactions $\mathcal{L}_{W \Phi}, \mathcal{L}_{B \Phi}$ and $\mathcal{L}_{W}$ are "contrived" [14 and "unnatural" [16], a few additional comments may be appropriate. As an example, we consider $\mathcal{L}_{W \Phi}$ as defined by (3.6). Identical arguments can be given for $\mathcal{L}_{B \Phi}$ and $\mathcal{L}_{W}$. The physical content of the theory described by the Lagrangian

$$
\mathcal{L}=\mathcal{L}_{S M}+\epsilon_{W \Phi} \frac{g}{M_{W}^{2}} \mathcal{L}_{W \Phi}
$$

remains invariant if the $W^{\mu}$ field is replaced by

$$
W_{i}^{\mu} \rightarrow W_{i}^{\mu}-\frac{i}{2} \epsilon_{W \Phi} \frac{g}{M_{W}^{2}} \operatorname{tr}\left[\Phi^{\dagger} \tau_{i}\left(D^{\mu} \Phi\right)\right]
$$

The invariance of the theory is due to the fact that a field transformation in the path integral does not change the theory'. To order $\epsilon_{W \Phi}$, the substitution (4.9) yields the Lagrangian

$$
\begin{aligned}
\mathcal{L}= & \mathcal{L}_{S M}+\epsilon_{W \Phi} \frac{g}{M_{W}^{2}}\left\{g^{\prime} \mathcal{L}_{W B}-g \mathcal{L}_{W W, 2}\right. \\
& \left.\left.-\frac{1}{8} g\left[\operatorname{tr}\left(\Phi^{\dagger} \tau_{i}\left(D_{\mu} \Phi\right)\right)\right]\left[\operatorname{tr}\left(\left(D^{\mu} \Phi^{\dagger}\right) \tau_{i} \Phi\right)-\operatorname{tr}\left(\Phi^{\dagger} \tau_{i}\left(D^{\mu} \Phi\right)\right)+2 i \bar{\Psi}_{L} \gamma^{\mu} \tau_{i} \Psi_{L}\right)\right]\right\} \\
& +O\left(\epsilon_{W \Phi}^{2}\right)+\delta^{4}(0) \text { terms }
\end{aligned}
$$

which is equivalent to (4.8), even though it looks rather complicated. Formula (4.10) and similar ones for $\mathcal{L}_{B \Phi}$ and $\mathcal{L}_{W}$ were given in [16].'.

While $\mathcal{L}_{W \Phi}$, according to its definition (3.6), only yields bosonic loop effects on LEP 1 observables, the form (4.10) of the Lagrangian now suddenly contains terms which, taken by themselves, contribute at tree level to LEP 1 observables, since they involve non-standard fermion interactions. One may explicitly convince oneself that the tree-level effects of (4.10) on LEP 1 observables cancel, as obviously implied by the equivalence of (4.8) and (4.10). The fact that the interactions $\mathcal{L}_{W B}$ and the fermionic term in (4.10), when individually analyzed, are strongly suppressed by the LEP 1 empirical results thus appears to be irrelevant for the strength and possible presence of $\mathcal{L}_{W \Phi}$. In other words, from the fact that certain fermion-boson terms are absent one can hardly deduce that also purely non-standard bosonic

\footnotetext{
${ }^{6}$ This holds apart from $\delta^{4}(0)$-terms, which arise from the Jacobian of the transformation.

${ }^{7}$ The fact that $(4.10$ is obtained by inserting the equations of motion [16 into the Lagrangian does not provide a sufficient justification for the validity of (4.10). Since the equations of motion must not be substituted into the Lagrangian, one has, instead, to perform the field transformation (4.9) to obtain the result (4.10) 29].
} 
interactions are absent in nature. Indeed, it is not a priori excluded that precisely the purely bosonic sector in which the non-Abelian structure of the gauge group and the spontaneous symmetry breaking via the Higgs mechanism are manifest deviates from orthodoxy. Direct empirical tests of the boson sector seem unavoidable. Moreover, we note that an interaction term, such as $\mathcal{L}_{W \Phi}(3.6)$, can hardly be considered as "unnatural" [16, simply because, by

a fairly involved field transformation, it can be cast into a lengthy and awkward form, such as $(4.10)$.

\subsection{Non-Standard Gauge-Boson Interactions}

In this section, we briefly collect the various standard and non-standard interactions implied by the three-parameter Lagrangian (4.2). We will use the parameters $\delta_{Z}, x_{\gamma}$ and $y_{Z}$, which are related to the couplings $\epsilon_{W \Phi}, \epsilon_{B \Phi}$ and $\epsilon_{W}$ by (3.12) and (4.3). We will restrict ourselves to the relevant trilinear and quadrilinear boson couplings (in the unitary gauge, $\varphi_{a} \equiv 0$ ). Multi-particle vertices of fairly remote significance are not listed.

\subsubsection{Trilinear Gauge-Boson Self-Interactions}

The trilinear couplings are parametrized as in (2.2). With (3.3) and (4.4) one finds in terms of $\delta_{Z}, x_{\gamma}$ and $y_{\gamma}$

$$
\begin{aligned}
\mathcal{L}_{3 G B}\left(\delta_{Z}, x_{\gamma}, y_{\gamma}\right)= & -i e\left[A_{\mu}\left(W^{-\mu \nu} W_{\nu}^{+}-W^{+\mu \nu} W_{\nu}^{-}\right)+A_{\mu \nu} W^{+\mu} W^{-\nu}\right] \\
& -i e\left(\frac{\cos \theta_{W}}{\sin \theta_{W}}+\delta_{Z}\right)\left[Z_{\mu}\left(W^{-\mu \nu} W_{\nu}^{+}-W^{+\mu \nu} W_{\nu}^{-}\right)+Z_{\mu \nu} W^{+\mu} W^{-\nu}\right] \\
& -i e x_{\gamma}\left(A_{\mu \nu} W^{+\mu} W^{-\nu}-\frac{\sin \theta_{W}}{\cos \theta_{W}} Z_{\mu \nu} W^{+\mu} W^{-\nu}\right) \\
& +i e \frac{y_{\gamma}}{M_{W}^{2}}\left(A_{\mu}{ }^{\nu} W_{\nu}^{-\lambda} W_{\lambda}^{+\mu}+\frac{\cos \theta_{W}}{\sin \theta_{W}} Z_{\mu}{ }^{\nu} W_{\nu}^{-\lambda} W_{\lambda}^{+\mu}\right)
\end{aligned}
$$

The three-gauge-boson interaction differs from the standard model in the overall normalization of the $Z^{0}$ coupling and in the non-standard dipole and quadrupole terms, in which the relative strengths of the $Z^{0}$ and $\gamma$ couplings are fixed, however.

\subsubsection{Quadrilinear Gauge-Boson Self-Interactions}

$\mathcal{L}_{B \Phi}$ does not contain quadrilinear gauge-boson self-interactions. Thus, the non-standard quadrilinear couplings only depend on $\epsilon_{W \Phi}$ and $\epsilon_{W}$ or, alternatively, by use of (3.12) and (4.3), on $\delta_{Z}$ and $y_{\gamma} . \mathcal{L}_{W \Phi}$ simply changes the coupling constants of the Yang-Mills interactions, while $\mathcal{L}_{W}$ yields extra couplings containing field strength tensors,

$$
\begin{aligned}
\mathcal{L}_{4 G B}\left(\delta_{Z}, y_{\gamma}\right)= & -e^{2}\left(A_{\mu} A^{\mu} W_{\nu}^{+} W^{-\nu}-A_{\mu} A_{\nu} W^{+\mu} W^{-\nu}\right) \\
& -2 e^{2} \frac{\cos \theta_{W}}{\sin \theta_{W}}\left(1+\delta_{Z} \frac{\sin \theta_{W}}{\cos \theta_{W}}\right) \\
& \left(A_{\mu} Z^{\mu} W_{\nu}^{+} W^{-\nu}-\frac{1}{2} A_{\mu} Z_{\nu}\left(W^{+\mu} W^{-\nu}+W^{-\mu} W^{+\nu}\right)\right) \\
& -e^{2} \frac{\cos ^{2} \theta_{W}}{\sin ^{2} \theta_{W}}\left(1+2 \delta_{Z} \frac{\sin \theta_{W}}{\cos \theta_{W}}\right)\left(Z_{\mu} Z^{\mu} W_{\nu}^{+} W^{-\nu}-Z_{\mu} Z_{\nu} W^{+\mu} W^{-\nu}\right) \\
& +e^{2} \frac{1}{2 \sin ^{2} \theta_{W}}\left(1+2 \delta_{Z} \sin \theta_{W} \cos \theta_{W}\right)
\end{aligned}
$$




$$
\begin{gathered}
\left(W_{\mu}^{+} W_{\nu}^{-} W^{+\mu} W^{-\nu}-W_{\mu}^{+} W_{\nu}^{-} W^{-\mu} W^{+\nu}\right) \\
+e^{2} \frac{y_{\gamma}}{M_{W}^{2}}\left\{\left[A_{\mu} A^{\mu \lambda}\left(W^{+\nu} W_{\nu \lambda}^{-}+W^{-\nu} W_{\nu \lambda}^{+}\right)\right.\right. \\
\left.+A_{\mu} A_{\nu \lambda}\left(W^{+\nu} W^{-\lambda \mu}+W^{-\nu} W^{+\lambda \mu}\right)\right] \\
+\frac{\cos \theta_{W}}{\sin \theta_{W}}\left[\left(A_{\mu} Z^{\mu \lambda}+Z_{\mu} A^{\mu \lambda}\right)\left(W^{+\nu} W_{\nu \lambda}^{-}+W^{-\nu} W_{\nu \lambda}^{+}\right)\right. \\
\left.\quad+\left(A_{\mu} Z_{\nu \lambda}+Z_{\mu} A_{\nu \lambda}\right)\left(W^{+\nu} W^{-\lambda \mu}+W^{-\nu} W^{+\lambda \mu}\right)\right] \\
+\frac{\cos ^{2} \theta_{W}}{\sin ^{2} \theta_{W}}\left[Z_{\mu} Z^{\mu \lambda}\left(W^{+\nu} W_{\nu \lambda}^{-}+W^{-\nu} W_{\nu \lambda}^{+}\right)\right. \\
\left.\quad+Z_{\mu} Z_{\nu \lambda}\left(W^{+\nu} W^{-\lambda \mu}+W^{-\nu} W^{+\lambda \mu}\right)\right] \\
\left.+\frac{1}{\sin ^{2} \theta_{W}}\left(W_{\mu}^{+} W^{+\mu \lambda} W^{-\nu} W_{\nu \lambda}^{-}-W_{\mu}^{+} W^{-\mu \lambda} W^{-\nu} W_{\nu \lambda}^{+}\right)\right\} .
\end{gathered}
$$

\subsubsection{Interactions of two Gauge Bosons and one or two Higgs Scalars}

$\mathcal{L}_{W}$ does not contain Higgs terms, i.e. the non-standard interactions involving the Higgs particle depend on $\delta_{Z}$ and $x_{\gamma}$ only. The interaction terms $\mathcal{L}_{W \Phi}$ and $\mathcal{L}_{B \Phi}$ extend the standard couplings of $W W H(H)$ and $Z Z H(H)$ by couplings involving one field-strength tensor and a derivative of the Higgs field, and they give rise to new $Z \gamma H(H)$ vertices in addition,

$$
\begin{aligned}
\mathcal{L}_{2 G B, 1 H}\left(\delta_{Z}, x_{\gamma}\right)= & e \frac{1}{\sin \theta_{W}} M_{W} W_{\mu}^{+} W^{-\mu} H+e \frac{1}{2 \sin \theta_{W} \cos ^{2} \theta_{W}} M_{W} Z_{\mu} Z^{\mu} H \\
& +e \frac{1}{M_{W}} \delta_{Z} \cos \theta_{W}\left(W_{\mu \nu}^{+} W^{-\mu}+W_{\mu \nu}^{-} W^{+\mu}\right) \partial^{\nu} H \\
& +e \frac{1}{M_{W}}\left(2 \delta_{Z} \sin \theta_{W}-x_{\gamma} \frac{1}{\cos \theta_{W}}\right) A_{\mu \nu} Z^{\mu} \partial^{\nu} H \\
& +e \frac{1}{M_{W}}\left(\delta_{Z}\left(\cos \theta_{W}-\frac{\sin ^{2} \theta_{W}}{\cos \theta_{W}}\right)+x_{\gamma} \frac{\sin \theta_{W}}{\cos ^{2} \theta_{W}}\right) Z_{\mu \nu} Z^{\mu} \partial^{\nu} H \\
\mathcal{L}_{2 G B, 2 H}= & e^{2} \frac{1}{4 \sin ^{2} \theta_{W}} M_{W} W_{\mu}^{+} W^{-\mu} H^{2}+e^{2} \frac{1}{8 \sin ^{2} \theta_{W} \cos ^{2} \theta_{W}} M_{W} Z_{\mu} Z^{\mu} H^{2} \\
+ & e \frac{1}{2 M_{W}} \delta_{Z} \frac{\cos \theta_{W}}{\sin \theta_{W}}\left(W_{\mu \nu}^{+} W^{-\mu}+W_{\mu \nu}^{-} W^{+\mu}\right)\left(\partial^{\nu} H\right) H \\
+ & +e^{2} \frac{1}{2 M_{W}^{2}}\left(2 \delta_{Z}-x_{\gamma} \frac{1}{\sin \theta_{W} \cos \theta_{W}}\right) A_{\mu \nu} Z^{\mu}\left(\partial^{\nu} H\right) H \\
& +e^{2} \frac{1}{2 M_{W}^{2}}\left(\delta_{Z}\left(\frac{\cos \theta_{W}}{\sin \theta_{W}}-\frac{\sin \theta_{W}}{\cos \theta_{W}}\right)+x_{\gamma} \frac{1}{\cos ^{2} \theta_{W}}\right) Z_{\mu \nu} Z^{\mu}\left(\partial^{\nu} H\right) H .
\end{aligned}
$$

\subsection{Leading Amplitudes for $\mathrm{e}^{+} \mathrm{e}^{-} \rightarrow \mathrm{W}^{+} \mathrm{W}^{-}$}

The effective Lagrangian (4.2) spoils the decent $\left(O\left(s^{0}\right)\right)$ high-energy behaviour of the treelevel helicity amplitudes for $e^{+} e^{-} \rightarrow W^{+} W^{-}$as given in the standard model. The helicity amplitudes for arbitrary parameters $\delta_{Z}, x_{\gamma}, x_{Z}, y_{\gamma}$ and $y_{Z}$ as defined in (2.2) may be found in [6] and [9]. Using the constraints (3.3), (4.4), we list the tree amplitudes to $O(\sqrt{s})$ and higher.

For equal transverse polarizations of the $W$ 's and lefthanded electrons the leading amplitude

$$
\mathcal{M}_{++}=\mathcal{M}_{--}=\frac{g^{2}}{8} \sin \vartheta \frac{s}{M_{W}^{2}} y_{\gamma}
$$


depends on the quadrupole term, only. For righthanded electrons the amplitude is $O\left(s^{0}\right)$. The amplitude for two $W$ 's with opposite transverse polarizations is also $O\left(s^{0}\right)$. The amplitude for two longitudinally polarized $W$ 's,

$$
\mathcal{M}_{L L}^{\sigma}=-\frac{e^{2}}{2} \sigma \sin \vartheta \frac{s}{M_{W}^{2}}\left[x_{\gamma}+\left(\frac{\sin \theta_{W}}{\cos \theta_{W}} x_{\gamma}-\delta_{Z}\right)(a-2 b \sigma)\right],
$$

gets no contribution from the quadrupole term and is determined by the modifications of the Yang-Mills couplings, $\delta_{Z}$ and $x_{\gamma}$. It has the same high energy behaviour as the amplitude (4.15) for equal transverse polarizations. For one longitudinally and one transversely polarized $W$ the amplitude

$$
\begin{aligned}
\mathcal{M}_{L T}=-\frac{e^{2}}{2 \sqrt{2}} \sigma(\tau \cos \vartheta-2 \sigma) \frac{\sqrt{s}}{M_{W}} & {\left[x_{\gamma}+y_{\gamma}+\left(x_{\gamma} \frac{\sin \theta_{W}}{\cos \theta_{W}}-2 \delta_{Z}-y_{\gamma} \frac{\cos \theta_{W}}{\sin \theta_{W}}\right)(a-2 b \sigma)\right], } \\
\text { with } \tau & =+1 \text { for } W^{+}(+) \text {or } W^{-}(-), \\
\tau & =-1 \text { for } W^{+}(-) \text {or } W^{-}(+),
\end{aligned}
$$

(where the signs in parentheses after the $W^{ \pm}$denote the helicities of the $W^{ \pm}$) is only $O(\sqrt{s})$. In the above formulae, $a$ and $b$ are the vector and axial couplings of the $Z^{0}$ given by

$$
a=\frac{1}{4 \sin \theta_{W} \cos \theta_{W}}\left(-1+4 \sin ^{2} \theta_{W}\right), \quad b=-\frac{1}{4 \sin \theta_{W} \cos \theta_{W}},
$$

$\sigma=-\frac{1}{2}, \frac{1}{2}$ for lefthanded and righthanded electrons, respectively, and $\vartheta$ is the center of mass scattering angle.

From (4.15) and (4.16) we conclude that even with the constraints (3.3) and (4.4) there is always at least one amplitude which grows as $O(s)$, unless all three parameters $\delta_{Z}, x_{\gamma}$ and $y_{\gamma}$ are simultaneously zero. Thus, the effects of the model we consider lead to a cross section for $e^{+} e^{-} \rightarrow W^{+} W^{-}$which grows as $s$ in the terms bilinear (quadratic) in $\delta_{Z}, x_{\gamma}$ and $y_{\gamma}$ and is $O\left(s^{0}\right)$ in the linear terms, while in the standard model the cross section decreases as $s^{-1}$. The sensitivity to non-standard couplings increases substantially with increasing energy.

\section{The Term $\mathcal{L}_{\mathrm{WB}}$}

The interaction term $\mathcal{L}_{W B}$ in (4.7) contains mixing in the neutral gauge-boson sector, $W_{\mu \nu}^{3} B^{\mu \nu}$, as well as non-standard trilinear interactions, $W_{\mu}^{+} W_{\nu}^{-} B^{\mu \nu}$, between the $B$ field and the charged vector bosons. It also contains non-standard Higgs-interactions. We will see that the Lagrangian resulting by adding $\mathcal{L}_{W B}$ to the standard Lagrangian, apart from the non-standard Higgs-interactions, coincides with the Hung-Sakurai model [17]. In other words, the term $\mathcal{L}_{W B}$ essentially yields an extension of this latter model which is invariant under local $\mathrm{SU}(2)_{\mathrm{L}} \times \mathrm{U}(1)_{\mathrm{Y}}$ transformations quite similar to the terms $\mathcal{L}_{W \Phi}$ and $\mathcal{L}_{B \Phi}$ which yield a locally invariant extension of the KMSS model.

\section{1 $\quad \mathbf{W}_{\mu \nu}^{3} \mathbf{B}^{\mu \nu}$ Mixing}

To start with, we concentrate on the $W_{\mu \nu}^{3} B^{\mu \nu}$ mixing term and the corresponding modification of the $W^{ \pm} Z^{0}$ mass relation and the fermionic couplings of the vector-bosons. In the 
Lagrangian

$$
\begin{aligned}
\mathcal{L}_{S M+W B} & =\mathcal{L}_{S M}+\epsilon_{W B} \frac{g^{2}}{M_{W}^{2}} \mathcal{L}_{W B}, \\
\text { where } \mathcal{L}_{W B} & =-\frac{1}{4} \operatorname{tr}\left(\Phi^{\dagger} W_{\mu \nu} \Phi \tau_{3}\right) B^{\mu \nu}
\end{aligned}
$$

we consider that part which describes the vector-boson-fermion interactions and the terms quadratic in the gauge-boson fields,

$$
\begin{aligned}
\mathcal{L}_{S M+W B, e f f}= & -\frac{1}{4} W_{\mu \nu}^{i} W_{i}^{\mu \nu}-\frac{1}{4} B_{\mu \nu} B^{\mu \nu}+M_{W}^{2} W_{\mu}^{+} W^{-\mu}-g W_{\mu}^{i} J_{i}^{\mu}-g^{\prime} B_{\mu} J_{Y}^{\mu} \\
& +\frac{1}{2} M_{W}^{2}\left(B W^{3}\right)^{\mu}\left(\begin{array}{cc}
\left(\frac{g^{\prime}}{g}\right)^{2} & -\frac{g^{\prime}}{g} \\
-\frac{g^{\prime}}{g} & 1
\end{array}\right)\left(\begin{array}{c}
B \\
W^{3}
\end{array}\right)_{\mu}-\frac{\epsilon_{W B}}{2} W_{\mu \nu}^{3} B^{\mu \nu} .
\end{aligned}
$$

The Lagrangian contains a mass mixing $\left(W_{3}^{\mu} B_{\mu}\right)$ term and a current-mixing $\left(W_{3}^{\mu \nu} B_{\mu \nu}\right)$ term. In (5.2), the basic parameters are $M_{W}, g, g^{\prime}$ and $\epsilon_{W B}$.

We will see that an appropriate transition to transformed fields, $W_{\mu \nu}^{\prime 3}$ and $B_{\mu \nu}^{\prime}$, as well as $\tilde{W}_{\mu \nu}^{3}$ and $\tilde{A}_{\mu \nu}$, allows one to rewrite the Lagrangian (5.2) in a form that contains either mass mixing only or current mixing only, respectively. In the current-mixing form the Lagrangian will be seen to coincide with the Hung-Sakurai Lagrangian.

One easily verifies by direct substitution that the transformation

$$
\left(\begin{array}{c}
B \\
W_{3}
\end{array}\right)=\left(\begin{array}{cc}
\frac{1}{\sqrt{1-\epsilon_{W B}^{2}}} & 0 \\
\frac{-\epsilon_{W B}}{\sqrt{1-\epsilon_{W B}^{2}}} & 1
\end{array}\right)\left(\begin{array}{c}
B^{\prime} \\
W_{3}^{\prime}
\end{array}\right)
$$

removes the current-mixing term in (5.2), modifies the mass matrix by terms that depend on $\epsilon_{W B}$ and introduces an interaction of the hypercharge field, $B_{\mu}^{\prime}$, with the third component of the weak isospin current, $J_{\mu}^{3}$, i.e., the neutral current part of (5.2) becomes

$$
\begin{aligned}
& \mathcal{L}_{N}=-\frac{1}{4} W_{\mu \nu}^{\prime 3} W_{3}^{\prime \mu \nu}-\frac{1}{4} B_{\mu \nu}^{\prime} B^{\prime \mu \nu} \\
& +\frac{1}{2} M_{W}^{2}\left(\begin{array}{ll}
B^{\prime} & W_{3}^{\prime}
\end{array}\right)^{\mu}\left(\begin{array}{cc}
\frac{\left(\frac{g^{\prime}}{g}+\epsilon_{W B}\right)^{2}}{1-\epsilon_{W B}^{2}} & -\frac{\frac{g^{\prime}}{g}+\epsilon_{W B}}{\sqrt{1-\epsilon_{W B}^{2}}} \\
-\frac{g^{\prime}}{\sqrt{1-\epsilon_{W B}^{2}}} & 1
\end{array}\right)\left(\begin{array}{c}
B^{\prime} \\
W^{\prime 3}
\end{array}\right)_{\mu} \\
& -g W_{3}^{\prime \mu} J_{\mu}^{3}-g^{\prime} B_{\mu}^{\prime}\left(-\frac{g}{g^{\prime}} \frac{\epsilon_{W B}}{\sqrt{1-\epsilon_{W B}}} J_{3}+\frac{1}{\sqrt{1-\epsilon_{W B}^{2}}} J_{Y}\right)^{\mu} .
\end{aligned}
$$

Now consider the Hung-Sakurai model [17]. In this model, global SU(2) weak isospin symmetry is broken by electromagnetism. This breaking is of the strength $\lambda$ and is implemented by a current-mixing term. There is no mass mixing; instead, the third component of the weak isospin $W$-triplet has the mass $M_{W}$, as required by global $\mathrm{SU}(2)$ invariance. The Hung-Sakurai Lagrangian is thus given by

$$
\begin{array}{r}
\mathcal{L}_{H . S .}=-\frac{1}{4} W_{\mu \nu}^{i} W_{i}^{\mu \nu}-\frac{1}{4} \tilde{F}_{\mu \nu} \tilde{F}^{\mu \nu}+\frac{1}{2} M_{W}^{2} W_{\mu}^{i} W_{i}^{\mu}-g J_{\mu}^{i} W_{i}^{\mu}-e J_{\mu}^{e . m .} \tilde{A}^{\mu}+\mathcal{L}_{m i x} \\
\mathcal{L}_{m i x}=-\frac{1}{2} \lambda \tilde{F}_{\mu \nu} \tilde{W}_{3}^{\mu \nu}, \quad W=\left(W_{1}, W_{2}, \tilde{W}_{3}\right)
\end{array}
$$


where

$$
J^{e . m .}=J^{3}+J^{Y} .
$$

To see the equivalence of the Lagrangians in (5.2), (5.4) with (5.5), the mass-mixing term in (5.4) has to be replaced by a current-mixing term of appropriate strength. The replacement is achieved by applying a transformation to the fields in (5.4) which coincides with the inverse of (5.3), apart from replacing $\epsilon_{W B}$ by a new parameter, $\lambda$, viz.,

$$
\left(\begin{array}{c}
B^{\prime} \\
W_{3}^{\prime}
\end{array}\right)=\left(\begin{array}{cc}
\sqrt{1-\lambda^{2}} & 0 \\
\lambda & 1
\end{array}\right)\left(\begin{array}{c}
\tilde{A} \\
\tilde{W}_{3}
\end{array}\right)
$$

The parameter $\lambda$ has to be chosen such that the mass mixing disappears and the primordial photon field, $\tilde{A}$, couples to the electromagnetic current. Upon substituting (5.7) into (5.4), one finds that indeed (5.4) is converted into the form (5.5), provided $\lambda$ is chosen as

$$
\lambda=\lambda\left(g, g^{\prime}, \epsilon_{W B}\right)=\frac{\frac{g^{\prime}}{g}+\epsilon_{W B}}{\sqrt{1+2 \frac{g^{\prime}}{g} \epsilon_{W B}+\left(\frac{g^{\prime}}{g}\right)^{2}}}
$$

and $e$ is identified with

$$
e=e\left(g, g^{\prime}, \epsilon_{W B}\right)=g^{\prime} \frac{\sqrt{1-\lambda^{2}}}{\sqrt{1-\epsilon_{W B}^{2}}} .
$$

Passing to $e, g, M_{W}$ and $\lambda$ as basic parameters of the theory and using (5.9) to eliminate $g^{\prime}$, we may express $\lambda$ as

$$
\lambda=\lambda\left(e, g, \epsilon_{W B}\right)=\frac{e}{g}\left(1-\epsilon_{W B}^{2}\right)+\epsilon_{W B} \sqrt{1-\left(\frac{e}{g}\right)^{2}\left(1-\epsilon_{W B}^{2}\right)} .
$$

Using this form of $\lambda$ allows one to represent the Hung-Sakurai Lagrangian (5.5) in terms of $e, g, M_{W}$ and the $B^{\mu \nu} W_{\mu \nu}^{3}$ current-mixing strength $\epsilon_{W B}$ in (5.2). We note the linear approximation of (5.10),

$$
\lambda=\frac{e}{g}+\epsilon_{W B} \sqrt{1-\frac{e^{2}}{g^{2}}}+O\left(\epsilon_{W B}^{2}\right)
$$

On the other hand,

$$
\lambda=\frac{e}{g}(1-\epsilon)
$$

where $\epsilon$ is identical to the parameter introduced in [18]. Thus

$$
\begin{aligned}
\epsilon & =-\epsilon_{W B}\left(\sqrt{\frac{g^{2}}{e^{2}}-1+\epsilon_{W B}^{2}}-\epsilon_{W B}\right) \\
& =-\epsilon_{W B} \frac{g}{e} \sqrt{1-\frac{e^{2}}{g^{2}}+O\left(\epsilon_{W B}^{2}\right) .}
\end{aligned}
$$

The parameter $-\epsilon$ coincides $[18$ with the non-standard contribution to the frequently used parameter $\epsilon_{3}$ [31]. 
The transition to the physical fields, i.e., simultaneous diagonalization of the mass and the kinetic term [30], may be carried out either by applying the transformation

$$
\left(\begin{array}{c}
B \\
W_{3}
\end{array}\right)=\left(\begin{array}{cc}
\sqrt{1-2 \lambda \frac{e}{g}+\left(\frac{e}{g}\right)^{2}} & -\frac{\sqrt{1-2 \lambda \frac{e}{g}+\left(\frac{e}{g}\right)^{2}} \lambda}{\sqrt{1-\lambda^{2}}} \\
\frac{e}{g} & \frac{1-\lambda \frac{e}{g}}{\sqrt{1-\lambda^{2}}}
\end{array}\right)\left(\begin{array}{c}
A \\
Z
\end{array}\right)
$$

to the Lagrangian (5.2) or by applying

$$
\left(\begin{array}{c}
\tilde{A} \\
\tilde{W}^{3}
\end{array}\right)=\left(\begin{array}{cc}
1 & -\frac{\lambda}{\sqrt{1-\lambda^{2}}} \\
0 & \frac{1}{\sqrt{1-\lambda^{2}}}
\end{array}\right)\left(\begin{array}{l}
A \\
Z
\end{array}\right)
$$

to the Lagrangian (5.5). One obtains (in the neutral sector)

$$
\begin{aligned}
\mathcal{L}_{N}= & -\frac{1}{4} Z_{\mu \nu} Z^{\mu \nu}+\frac{1}{2} \frac{M_{W}^{2}}{1-\lambda^{2}} Z_{\mu} Z^{\mu} \\
& -\frac{1}{4} F_{\mu \nu} F^{\mu \nu}-\frac{g}{\sqrt{1-\lambda^{2}}}\left(J^{3}-\lambda \frac{e}{g} J^{e . m \cdot}\right)^{\mu} Z_{\mu}-e J_{\mu}^{e . m \cdot} A^{\mu} .
\end{aligned}
$$

Clearly, (5.16) differs from the standard theory by the parameter $\lambda$, i.e., we have $M_{W}, e, g$ and $\lambda\left(e, g, \epsilon_{W B}\right)$ as basic parameters.

From (5.16), one reads off that $\sin ^{2} \theta_{W}$, defined as the negative ratio of the coefficients of $J^{e . m .}$ and $J^{3}$ in (5.16), is in this model given by

$$
\sin ^{2} \theta_{W}=\lambda \frac{e}{g}
$$

while the mass of the $Z^{0}$ turns out to be

$$
M_{Z}^{2}=\frac{M_{W}^{2}}{1-\lambda^{2}}
$$

Introducing the Fermi coupling, $G_{F}$,

$$
g^{2}=\frac{8 M_{W}^{2} G_{F}}{\sqrt{2}}
$$

and using (5.17) and (5.18), we may replace the input parameters $e, g, \lambda$ and $M_{W}$ by the set $\alpha, G_{F}, M_{Z}$ and $M_{W}$. One obtains

$$
\sin ^{2} \theta_{W}=\sqrt{\frac{\pi \alpha}{\sqrt{2} G_{F} M_{Z}^{2}}\left(\frac{M_{Z}^{2}}{M_{W}^{2}}-1\right)},
$$

or, by solving for $M_{W}^{2}$,

$$
M_{W}^{2}=\frac{M_{Z}^{2}}{1+\frac{\sqrt{2} G_{F} M_{Z}^{2} \sin ^{2} \theta_{W}}{\alpha \pi}} .
$$

\footnotetext{
${ }^{8}$ This effective Lagrangian is the same as the one obtained (in a certain limit) from a theory with an additional vector-boson triplet, $V^{ \pm}, V^{0}$, associated with an $S U(2)_{V}$ local symmetry [18, 32].
} 
The relation between $\epsilon_{W B}$ and the set $\alpha, G_{F}, M_{Z}$ and $M_{W}$ is contained in (5.18), (5.10) and (5.19). By solving for $M_{W}^{2}$ one obtains

$$
\frac{M_{W}^{2}}{M_{Z}^{2}}=\frac{1-\epsilon_{W B}^{2}-2 B \epsilon_{W B} \sqrt{1-\epsilon_{W B}^{2}}}{2}+\left(1-\epsilon_{W B}^{2}\right) \sqrt{\frac{1}{4}-B^{2}-\frac{B \epsilon_{W B}}{\sqrt{1-\epsilon_{W B}^{2}}}},
$$

where $B$ is defined by

$$
B \equiv \sqrt{\frac{\pi \alpha}{\sqrt{2} G_{F} M_{Z}^{2}}}
$$

For small $\epsilon_{W B}$ we have

$$
\frac{M_{W}^{2}}{M_{Z}^{2}}=\frac{1}{2}+\sqrt{\frac{1}{4}-B^{2}}-B\left(1+\frac{1}{\sqrt{1-4 B^{2}}}\right) \epsilon_{W B}+O\left(\epsilon_{W B}^{2}\right) .
$$

The parameter $\epsilon$ introduced in (5.12) may be written as a function of $\sin ^{2} \theta_{W}$,

$$
\epsilon\left(\sin ^{2} \theta_{W}\right)=1-\frac{\sin ^{2} \theta_{W} B^{-2}}{1+\sin ^{4} \theta_{W} B^{-2}},
$$

or, alternatively, as a function of $M_{W}$,

$$
\epsilon\left(M_{W}\right)=1-\sqrt{\frac{\sqrt{2} G_{F} M_{W}^{2}}{\alpha \pi}\left(1-\frac{M_{W}^{2}}{M_{Z}^{2}}\right)},
$$

and (5.13) becomes

$$
\epsilon_{W B}=-\epsilon \frac{\sin \theta_{W}}{\sqrt{1-\sin ^{2} \theta_{W}}}+O\left(\epsilon^{2}\right)
$$

\subsection{Observability of $\mathcal{L}_{\mathrm{WB}}$}

When deducing $\epsilon$ from the empirical data on the W-mass, the LEP 1 and the low- $q^{2}$ data, radiative corrections have to be taken into account. The dependence of the radiative corrections on the (unknown) value of the top-quark mass, $m_{t}$, implies a dependence on $m_{t}$ 《4 of the value of $\epsilon$ deduced from the data. A careful two-parameter fit 18 gave the result

$$
\begin{aligned}
\epsilon & =(0.48 \pm 0.61) \cdot 10^{-2} \\
m_{t} & =(114 \pm 32) \mathrm{GeV}
\end{aligned}
$$

This result was based on a fit to the combined LEP 1 data [33], the W-mass measurement [34], and the low $q^{2}$ data [35]. A new evaluation based on the most recent LEP 1 data will significantly diminish the error in the determination of $\epsilon$. Such a fit will be presented elsewhere.

In the present paper, we will restrict ourselves to a brief discussion of the dependence of the error in the determination of $\epsilon$ resulting from the experimental errors in $M_{W}$ and $\sin ^{2} \theta_{W}$ keeping $m_{t}$ fixed.

When deducing $\epsilon$ from a measurement of the W-mass, one finds from (5.26) for the error, $\Delta \epsilon$, of $\epsilon$

$$
\Delta \epsilon\left(M_{W}\right)=\frac{c^{2}-s^{2}}{s^{2} c} \frac{\Delta M_{W}}{\sqrt{\rho} M_{Z}}+O(\epsilon \Delta \epsilon) \simeq 0.0289 \mathrm{GeV}^{-1} \Delta M_{W}
$$


where $s^{2}=1-c^{2}$ is the standard weak angle evaluated with the replacements (e.g. [4])

$$
\alpha(0) \rightarrow \alpha\left(M_{Z}^{2}\right) \simeq \frac{1}{128.9}
$$

and

$$
M_{Z}^{2} \rightarrow \rho M_{Z}^{2}
$$

i.e., $s^{2}$ is obtained from

$$
s^{2} c^{2}=\frac{\pi \alpha\left(M_{Z}^{2}\right)}{\sqrt{2} G_{F} \rho M_{Z}^{2}} \equiv \tilde{B}^{2}
$$

by solving for $s^{2}$,

$$
s^{2}=\frac{1}{2}-\sqrt{\frac{1}{4}-\tilde{B}^{2}} .
$$

The replacements (5.30) and (5.31) take into account radiative corrections with sufficient accuracy for the present purpose of estimating the accuracy with which $\epsilon$ can be determined from the data. A dependence on $m_{t}$ enters in (5.29) via the $m_{t}$-dependence of the $\rho$ parameter (e.g., [4]). The value of $\Delta \epsilon$, however, changes by less than $4 \%$ if $m_{t}$ is varied from $100 \mathrm{GeV}$ to $200 \mathrm{GeV}$. The numerical evaluation of (5.29), using the present error in the W-mass measurement [34],

$$
M_{W}=80.13 \pm 0.27 \mathrm{GeV}
$$

and the accuracy in $M_{W}$ of $\Delta M_{W}=60 \mathrm{GeV}$ to be expected (e.g., [36]) from LEP 2, yields, respectively,

$$
\Delta \epsilon\left(M_{W}\right)=\left\{\begin{array}{lll}
0.78 \cdot 10^{-2} & \text { for } & \Delta M_{W}=270 \mathrm{GeV} \\
0.17 \cdot 10^{-2} & \text { for } & \Delta M_{W}=60 \mathrm{GeV}
\end{array}\right.
$$

The corresponding value of $\epsilon_{W B}$, according to (5.27), is given by

$$
\Delta \epsilon_{W B} \simeq-0.55 \Delta \epsilon .
$$

In an analogous manner, from (5.25), we obtain for $\Delta \epsilon$ as determined from the measurement of $\sin ^{2} \theta_{W}$,

$$
\Delta \epsilon\left(\sin ^{2} \theta_{W}\right)=-\frac{c^{2}-s^{2}}{s^{2}} \Delta \sin ^{2} \theta_{W}+O(\epsilon \Delta \epsilon) \simeq-2.31 \Delta \sin ^{2} \theta_{W} .
$$

Here, $\sin ^{2} \theta_{W}$ is the weak mixing angle at the $\mathrm{Z}^{0}$-mass obtained from the vector and axial vector couplings of the $\mathrm{Z}^{0}$ to leptons, i.e.,

$$
\sin ^{2} \theta_{W} \equiv \bar{s}_{W}^{2}\left(M_{Z}^{2}\right)=\frac{1}{4}\left(1-\frac{g_{V}^{l}}{g_{A}^{l}}\right) .
$$

Inserting for $\Delta \bar{s}_{W}^{2}$ the error deduced from the charged lepton forward-backward asymmetries, using the LEP 1 results given in [33] and the most recent ones given in [37], as well as the future accuracy expected by combining LEP 1 and SLC data [1], we obtain, respectively,

$$
\Delta \epsilon\left(\sin ^{2} \theta_{W}\right)=\left\{\begin{array}{l}
0.72 \cdot 10^{-2} \text { for } \Delta \bar{s}_{W}^{2}=0.0031\left(\text { from } A_{F B}^{l}=0.0138 \pm 0.0049\right)[33], \\
0.28 \cdot 10^{-2} \text { for } \Delta \bar{s}_{W}^{2}=0.0012\left(\text { from } A_{F B}^{l}=0.0164 \pm 0.0021\right) \text { [37], } \\
0.09 \cdot 10^{-2} \text { for } \Delta \bar{s}_{W}^{2}=0.0004 \text { [1]. }
\end{array}\right.
$$

With the assumption that the value of $\epsilon$ obtained from a careful fit of the available data will allow for $\epsilon=0$ to lie within the error bars given by (5.35) and (5.39), the strength of a possible $\mathrm{SU}(2)_{\mathrm{L}}$ violation in vector-boson fermion couplings, $\epsilon \cdot e$ [1], will be restricted in magnitude to a value of the order of $0.2 \cdot 10^{-2} e$. 


\subsection{Trilinear Gauge-Boson Self-Interactions Induced by $\mathcal{L}_{\mathrm{WB}}$}

The term $\mathcal{L}_{W B}$ has direct and indirect effects on the self-couplings, leading to nonstandard values of $g_{Z W W}, \kappa_{\gamma}$ and $\kappa_{Z}$. The direct effects are due to the trilinear interactions $B_{\mu \nu} W^{+\mu} W^{-\nu}$ contained in $\mathcal{L}_{W B}$. The indirect effects are due to mixing between $W_{3}^{\mu}$ and $B^{\mu}$, which also affects the self-couplings in $\mathcal{L}_{S M}$. With $\delta_{Z}$ being defined as the deviation from the standard case, viz.,

$$
\begin{aligned}
\delta_{Z} & \equiv g_{Z W W}(e, g, \lambda)-g_{Z W W}\left(e, g, \lambda=\frac{e}{g}\right) \\
& =g_{Z W W}(e, g, \lambda)-\sqrt{\left(\frac{g}{e}\right)^{2}-1}
\end{aligned}
$$

one finds

$$
\begin{aligned}
\delta_{Z} & =\frac{g}{e \sqrt{1-\lambda^{2}}}\left(1-\lambda \frac{e}{g}\right)-\sqrt{\left(\frac{g}{e}\right)^{2}-1}, \\
x_{\gamma} & =\lambda \frac{g}{e}-1, \\
x_{Z} & =-\frac{\lambda}{\sqrt{1-\lambda^{2}}}\left(\lambda \frac{g}{e}-1\right),
\end{aligned}
$$

i.e., using (5.18), $x_{Z}$ is given in terms of $x_{\gamma}$ by

$$
x_{Z}=-x_{\gamma} \sqrt{\left(\frac{M_{Z}}{M_{W}}\right)^{2}-1} .
$$

Such a proportionality of $x_{Z}$ to $x_{\gamma}$ is expected, as intrinsic $\mathrm{SU}(2)_{\mathrm{L}}$ violation (of the form (3.4)) is absent in $\mathcal{L}_{W B}$. Also passing to the basic input parameters $\alpha, G_{F}, M_{Z}$ and $M_{W}$ in $\delta_{Z}$ and $x_{\gamma}$, we have

$$
\begin{aligned}
& \delta_{Z}=\frac{1}{\sin ^{2} \theta_{W}}\left[\cos ^{2} \theta_{W} \sqrt{\left(\frac{M_{Z}}{M_{W}}\right)^{2}-1}-\sqrt{1-\left(\frac{M_{W}}{M_{Z}}\right)^{2}-\sin ^{4} \theta_{W}}\right] \\
& x_{\gamma}=\frac{1}{\sin ^{2} \theta_{W}}\left(1-\left(\frac{M_{W}}{M_{Z}}\right)^{2}\right)-1,
\end{aligned}
$$

where $\sin ^{2} \theta_{W}$ in terms of $\alpha, G_{F}, M_{Z}$ and $M_{W}$ is given by (5.20). Alternatively, by substituting $M_{W}$ from (5.21), one may express (5.42), (5.43) in terms of $\alpha, G_{F}, M_{Z}$ and $\sin ^{2} \theta_{W}$ as basic parameters. Note that all parameters in (5.42), (5.43) can be measured independently of observing $e^{+} e^{-} \rightarrow W^{+} W^{-}$. Obviously, the standard model self-interactions, $\delta_{Z}=x_{\gamma}=x_{Z}=0$, are recovered if the standard relation

$$
\sin ^{2} \theta_{W}=1-\frac{M_{W}^{2}}{M_{Z}^{2}}
$$

is fulfilled. In this case, with (5.20), (5.21) $M_{W}$ and $\sin ^{2} \theta_{W}$ are determined by $\alpha, G_{F}$ and $M_{Z}$ alone.

We note that also the vector-boson self-interactions (5.42) and (5.43) following from the Lagrangian (5.1) are the same as those obtained in the Hung-Sakurai model [17]. There, the vector-boson self-interactions are introduced by replacing the field-strength tensor $W_{\mu \nu}$ in (5.5) by the non-Abelian one and then coupling the primordial photon field, $\tilde{A}^{\mu}$, minimally 
to the $W^{\mu}$ field via replacing the derivatives on the $W$ field by covariant ones with respect to the electromagnetic gauge freedom.

Also with respect to the quadrilinear vector-boson self-interactions, this minimal substitution procedure of the Hung-Sakurai model leads to the same results as implied by the Lagrangian (5.1).

\subsection{Effects of $\mathcal{L}_{\mathrm{WB}}$ on $\mathrm{e}^{+} \mathrm{e}^{-} \rightarrow \mathrm{W}^{+} \mathrm{W}^{-}$}

The modifications in the trilinear couplings of the vector bosons among each other and of the vector bosons to the fermions caused by the presence of $\mathcal{L}_{W B}$ lead to deviations from the standard model predictions in the high-energy behaviour of the amplitudes for the process $e^{+} e^{-} \rightarrow W^{+} W^{-}$. The deviations are fairly modest, however. The high-energy behaviour of the helicity amplitudes, both for the production ot two longitudinal and of two transverse Wbosons, is the same as in the standard case, i.e., $O\left(s^{0}\right)$. This decent high-energy behaviour in the presence of the non-standard couplings (5.42) and (5.43) is due to cancellations among the non-standard effects arising from the fermion-vector-boson and the trilinear bosonic vertices. The only deviation from the standard-model high-energy behaviour occurs in the amplitudes for the production of one longitudinal and one transverse W-boson which grow as $\sqrt{s}$ and, for $s \gg 4 M_{W}^{2}$, are given by

$$
\mathcal{M}_{L T}(\sigma, \tau, q)=\frac{e^{2}}{2 \sqrt{2}} \frac{\sqrt{s} M_{Z}^{2}}{M_{W}^{2}} F(\sigma)(q-\sigma \tau \cos \vartheta)
$$

where

$$
\begin{aligned}
F(1) & =\lambda \frac{g}{e}-1=-\epsilon \\
F(-1) & =\frac{3}{2} \lambda \frac{g}{e}-1-\frac{1}{2}\left(\lambda \frac{g}{e}\right)^{2}=-\frac{\epsilon}{2}+O\left(\epsilon^{2}\right) .
\end{aligned}
$$

In (5.45), $\sigma=-1$ and $\sigma=+1$ refer to lefthanded and righthanded electrons, respectively. The helicity of the transverse $\mathrm{W}^{ \pm}$is denoted by $\tau= \pm 1$. Finally, the amplitude also depends on which one of the two W-bosons, the one with charge $q=+1$ or the one with charge $q=-1$, is in the transverse polarization state. As the amplitude in (5.45) is the only one with non-standard high-energy behaviour, and as the growth with $\sqrt{s}$ is a modest one, a determination of $\epsilon$ (or, equivalently, $\epsilon_{W B}$,) from the non-standard high-energy behaviour will, at LEP 2 or NLC energies, not reach the above-mentioned precision based on measurements of $\sin ^{2} \theta_{W}$ and the W-mass.

We note that the good high-energy behaviour of the model with $\mathcal{L}_{W B}$ also follows from its equivalence (apart from the Higgs-interactions) to the Hung-Sakurai model. This latter model is known [17 to have good high-energy behaviour apart from the $\sqrt{s}$ growth of the longitudinal-transverse amplitude in (5.45). The fairly good high-energy behaviour, accordingly, cannot be traced back to an underlying $\mathrm{SU}(2)_{\mathrm{L}} \times \mathrm{U}(1)_{\mathrm{Y}}$ gauge invariance (in disagreement with a statement in [16]), as this invariance is violated in the Hung-Sakurai model. In fact, it is stressed by these latter authors that the absence of $O(s)$ terms even holds when, as a consequence of $\lambda \neq e / g, \mathrm{SU}(2)_{\mathrm{L}} \times \mathrm{U}(1)_{\mathrm{Y}}$ symmetry is violated. It is true, however, that any departure from (5.41), (5.42) leads to a considerably stronger growth [16] of the high-energy helicity amplitudes than shown in (5.45). 


\subsection{Gauge-Boson Self-Interactions in the Full Model}

We turn to a brief analysis of the full Lagrangian

$$
\mathcal{L}_{e f f}=\mathcal{L}_{S M}+\left(\epsilon_{W} \frac{g}{M_{W}^{2}} \mathcal{L}_{W}+\epsilon_{W \Phi} \frac{g}{M_{W}^{2}} \mathcal{L}_{W \Phi}+\epsilon_{B \Phi} \frac{g^{\prime}}{M_{W}^{2}} \mathcal{L}_{B \Phi}+\epsilon_{W B} \frac{g^{2}}{M_{W}^{2}} \mathcal{L}_{W B}\right)
$$

which includes $W^{3} B$ mixing.

\subsubsection{Trilinear Gauge-Boson Self-Interactions}

It will be convenient to use $\lambda\left(e, g, \epsilon_{W B}\right)$ from (5.10) instead of $\epsilon_{W B}$ when giving the expressions for the trilinear couplings. In terms of $e, g, \lambda\left(e, g, \epsilon_{W B}\right)$ and $\epsilon_{B \Phi}, \epsilon_{W \Phi}$ and $\epsilon_{W}$, one finds

$$
\begin{aligned}
\delta_{Z} & =\frac{g}{e \sqrt{1-\lambda^{2}}}\left(1-\lambda \frac{e}{g}+\epsilon_{W \Phi}\right)-\sqrt{\left(\frac{g}{e}\right)^{2}-1} \\
x_{\gamma} & =\lambda \frac{g}{e}-1+\epsilon_{W \Phi}+\epsilon_{B \Phi} \\
x_{Z} & =-\frac{\lambda}{\sqrt{1-\lambda^{2}}}\left(\lambda \frac{g}{e}-1+\epsilon_{W \Phi}+\epsilon_{B \Phi}\right) \\
y_{\gamma} & =\epsilon_{W} \\
y_{Z} & =\epsilon_{W} \frac{g}{e} \frac{1-\lambda \frac{e}{g}}{\sqrt{1-\lambda^{2}}} .
\end{aligned}
$$

One immediately convinces oneself that the non-standard couplings previously given in (3.12), (3.13) and (4.3), (4.4) are recovered from (5.48) in the limit of $\lambda=e / g=\sin \theta_{W}$ which corresponds to $\epsilon_{W B}=0$ (compare (5.10) and (5.17)). Even for $\lambda \neq e / g\left(\epsilon_{W B} \neq 0\right)$, however, from (5.48), one immediately sees that $x_{Z}$ is proportional to $x_{\gamma}$, while $y_{Z}$ is proportional to $y_{\gamma}$. The proportionality of these couplings, previously (for $\epsilon_{W B}=0$ ) given in (3.13) and (4.4), thus remains valid even in the presence of the addition of $\mathcal{L}_{W B}$ in (5.47). Note that this result (which is not based on any approximation) is non-trivial insofar as $\mathcal{L}_{W B}$ contains $B W_{3}$ mixing as well as direct non-standard $B W^{+} W^{-}$couplings. Using (5.17) and (5.18) the proportionality relations contained in (5.48) become

$$
\begin{aligned}
x_{Z} & =-x_{\gamma} \sqrt{\left(\frac{M_{Z}}{M_{W}}\right)^{2}-1} \\
y_{Z} & =y_{\gamma} \frac{\cos ^{2} \theta_{W}}{\sin ^{2} \theta_{W}} \sqrt{\left(\frac{M_{Z}}{M_{W}}\right)^{2}-1 .}
\end{aligned}
$$

They only differ from the case of $\epsilon_{W B}=0$ in the values of the proportionality constants which in the present case depend on $\alpha, G_{F}, M_{Z}$ and a fourth free parameter, e.g., $M_{W}$ (compare (5.22) ) or $\sin ^{2} \theta_{W}$ (compare (5.20)). As $\alpha, G_{F}, M_{Z}$ as well as $M_{W}$ can be determined independently of a measurement of the couplings of the vector bosons to each other, and as $\epsilon_{W B} \neq 0$ only changes the magnitude of the factors in (5.49), the presence or absence of $\mathcal{L}_{W B}$ in (5.47) is fairly irrelevant for the phenomenology of the couplings among the vector bosons. Quite independently of the presence or absence of $\epsilon_{W B}$, there are three independent trilinear couplings, $\delta_{Z}, x_{\gamma}$ and $y_{\gamma}$, while $x_{Z}$ and $y_{Z}$ are proportional to $x_{\gamma}$ and $y_{\gamma}$, respectively. This conclusion is quantitavely, but not qualitatively, changed by the fact that actually $\epsilon_{W B}$ is strongly constrained anyway (compare (5.28) ) by LEP 1 measurements. 


\subsubsection{Quadrilinear Gauge-Boson Self-Interactions}

The above argument goes through for quadrilinear gauge-boson self-interactions as well. This is, however, not so surprising, as the presence of $\mathcal{L}_{W B}$ has only indirect influence on these couplings, in distinction from the case of the trilinear couplings. The couplings given by (1.12) can be expressed in terms of the two free parameters $\epsilon_{W \Phi}$ and $\epsilon_{W}$, and they generalize in the full model to

$$
\begin{aligned}
& \mathcal{L}_{4 G B}\left(\lambda, \epsilon_{W \Phi}, \epsilon_{W}\right)=-e^{2}\left(A_{\mu} A^{\mu} W_{\nu}^{+} W^{-\nu}-A_{\mu} A_{\nu} W^{+\mu} W^{-\nu}\right) \\
& -2 e^{2} \sqrt{\frac{M_{Z}^{2}}{M_{W}^{2}}-1} \frac{\cos ^{2} \theta_{W}}{\sin ^{2} \theta_{W}}\left(1+\frac{\epsilon_{W \Phi}}{\cos ^{2} \theta_{W}}\right) \\
& \left(A_{\mu} Z^{\mu} W_{\nu}^{+} W^{-\nu}-\frac{1}{2} A_{\mu} Z_{\nu}\left(W^{+\mu} W^{-\nu}+W^{-\mu} W^{+\nu}\right)\right) \\
& -e^{2}\left(\frac{M_{Z}^{2}}{M_{W}^{2}}-1\right) \frac{\cos ^{4} \theta_{W}}{\sin ^{4} \theta_{W}}\left(1+2 \frac{\epsilon_{W \Phi}}{\cos ^{2} \theta_{W}}\right) \\
& \left(Z_{\mu} Z^{\mu} W_{\nu}^{+} W^{-\nu}-Z_{\mu} Z_{\nu} W^{+\mu} W^{-\nu}\right) \\
& +\frac{g^{2}}{2}\left(1+2 \epsilon_{W \Phi}\right)\left(W_{\mu}^{+} W_{\nu}^{-} W^{+\mu} W^{-\nu}-W_{\mu}^{+} W_{\nu}^{-} W^{-\mu} W^{+\nu}\right) \\
& +e^{2} \frac{\epsilon_{W}}{M_{W}^{2}}\left\{\left[A_{\mu} A^{\mu \lambda}\left(W^{+\nu} W_{\nu \lambda}^{-}+W^{-\nu} W_{\nu \lambda}^{+}\right)\right.\right. \\
& \left.+A_{\mu} A_{\nu \lambda}\left(W^{+\nu} W^{-\lambda \mu}+W^{-\nu} W^{+\lambda \mu}\right)\right] \\
& +\sqrt{\frac{M_{Z}^{2}}{M_{W}^{2}}-1} \frac{\cos ^{2} \theta_{W}}{\sin ^{2} \theta_{W}}\left[\left(A_{\mu} Z^{\mu \lambda}+Z_{\mu} A^{\mu \lambda}\right)\left(W^{+\nu} W_{\nu \lambda}^{-}+W^{-\nu} W_{\nu \lambda}^{+}\right)\right. \\
& \left.+\left(A_{\mu} Z_{\nu \lambda}+Z_{\mu} A_{\nu \lambda}\right)\left(W^{+\nu} W^{-\lambda \mu}+W^{-\nu} W^{+\lambda \mu}\right)\right] \\
& +\left(\frac{M_{Z}^{2}}{M_{W}^{2}}-1\right) \frac{\cos ^{4} \theta_{W}}{\sin ^{4} \theta_{W}}\left[Z_{\mu} Z^{\mu \lambda}\left(W^{+\nu} W_{\nu \lambda}^{-}+W^{-\nu} W_{\nu \lambda}^{+}\right)\right. \\
& \left.\left.+Z_{\mu} Z_{\nu \lambda}\left(W^{+\nu} W^{-\lambda \mu}+W^{-\nu} W^{+\lambda \mu}\right)\right]\right\} \\
& +g^{2} \frac{\epsilon_{W}}{M_{W}^{2}}\left(W_{\mu}^{+} W^{+\mu \lambda} W^{-\nu} W_{\nu \lambda}^{-}-W_{\mu}^{+} W^{-\mu \lambda} W^{-\nu} W_{\nu \lambda}^{+}\right) .
\end{aligned}
$$

Again, the modifications due to arbitrary $\lambda$ only become manifest in the mismatch between $1-\sin ^{2} \theta_{W}$ and the mass ratio.

\section{Conclusion}

A priori, upon excluding C- and P-violation, there exist three dimension-four and two dimension-six trilinear couplings among the electroweak vector bosons, and there exists a large number of quadrilinear couplings.

As is well-known, via the minimum requirement that global $S U(2)$ symmetry only be broken by electromagnetism, the number of dimension-four couplings is reduced from three to two by relating the weak and electromagnetic dipole moments of the $W^{ \pm}$to each other. At the expense of introducing dimension-six terms with non-standard Higgs-boson couplings, we have shown that the above two-parameter dimension-four interactions can be embedded into a spontaneously broken $S U(2)_{L} \times U(1)_{Y}$ theory. This embedding is irrelevant for the phenomenological exploration of vector-boson self-couplings by direct measurements of vector-boson production in $e^{+} e^{-}$annihilation. The inclusion of non-standard Higgs interactions assures decent one-loop corrections to LEP 1 observables, however, and, accordingly, 
serves as an example of how the above two-parameter model for trilinear and quadrilinear boson couplings can be made consistent with LEP 1 empirical results.

The trilinear quadrupole couplings, in their well-known single-parameter form, are invariant under local $S U(2)_{L} \times U(1)_{Y}$ transformations and, accordingly, there is no need for non-standard Higgs terms.

The standard $S U(2)_{L} \times U(1)_{Y}$ theory with its celebrated renormalization properties obviously constitutes the simplest extension of our present empirical knowledge with respect to the empirically unknown trilinear and quadrilinear interactions of the vector bosons with one another. Good arguments may indeed be put forward for the realization of these standard interactions in nature. The dimension-six three-parameter alternative of the present paper is to be considered as an example of how non-standard (trilinear) couplings, only restricted by a minimum amount of $\mathrm{SU}(2)$ symmetry, can nevertheless coexist with LEP 1 precision data. Direct measurements of these couplings will be indispensable, if one desires to fully reveal the nature of the electroweak vector bosons.

\section{Acknowledgement}

It is a pleasure to thank M. Bilenky, W. Hollik, F. M. Renard and H. Spiesberger for useful discussions.

\section{References}

[1] L. Rolandi, Talk given on the XXVI ICHEP conference 1992 (Dallas), CERN- Preprint CERN-PPE/92-175 (1992)

[2] S. L. Glashow, Nucl. Phys. 22 (1961) 579;

S. Weinberg, Phys. Rev. Lett. 19 (1967) 1264;

A. Salam, Proc. 8th Nobel Symposium, ed. N. Svartholm (Almquits and Wiksells, Stockholm, 1968), p. 367

[3] G. J. Gounaris and D. Schildknecht, Z. Phys. C40 (1988) 477;

[4] J.-L. Kneur, M. Kuroda and D. Schildknecht, Phys. Lett. B262 (1991) 93

[5] Proc. of the ECFA Workshop on LEP 200, A. Böhm, W. Hoogland eds., CERN 87-08 (1987)

[6] M. Bilenky, J. L. Kneur, F. M. Renard and D. Schildknecht, Bielefeld-Preprint BI-TP $92 / 44$, to appear in Nucl. Phys. B

and literature cited there

[7] C. Grosse-Knetter and D. Schildknecht, Phys. Lett. B302 (1993) 309

[8] G. Bélanger and F. Boudjema, in " $e^{+} e^{-}$Collisions at $500 \mathrm{GeV}$, The Physics Potential", ed. P. W. Zerwas, DESY-Preprint DESY 92-123 (1992), p. 763

[9] G. Gounaris, J. L. Kneur, J. Layssac, G. Moultaka, F. M. Renard and D. Schildknecht, in " $e^{+} e^{-}$Collisions at $500 \mathrm{GeV}$, The Physics Potential", ed. P. W. Zerwas, DESYPreprint DESY 92-123 (1992), p. 735;

M. Bilenky, J.-L. Kneur, F. M. Renard and D. Schildknecht, in preparation 
[10] M. Frank, P. Mättig, R. Settles and W. Zeuner in " $e^{+} e^{-}$Collisions at $500 \mathrm{GeV}$, The Physics Potential”, ed. P. W. Zerwas, DESY-Preprint DESY 92-123 (1992), p. 223;

T. L. Barklow in "Proc. of the Workshop on Physics and Experiments with Linear Colliders", Saariselkä, Finnland, 1991 (World Scientific 1992), eds. P. Eerola et al.

[11] J. Maalampi, D. Schildknecht and K. H. Schwarzer, Phys. Lett. B166 (1986) 361; M. Kuroda, J. Maalampi, D. Schildknecht and K. H. Schwarzer, Nucl. Phys. B284 (1987) 271;

M. Kuroda, J. Maalampi, D. Schildknecht and K. H. Schwarzer, Phys. Lett. B190 (1987) 217

[12] D. Schildknecht, in Proc. 6th Topical Workshop on $p \bar{p}$-Collider Physics, Aaachen, ed.

K. Eggert, H. Faissner and E. Rademacher (World Scietific, 1986) p. 125

[13] K. Hagiwara, S. Ishihara, R. Szalapski and D. Zeppenfeld, Phys. Lett. B283 (1992) 353

[14] P. Hernández and F. J. Vegas, Phys. Lett. B307 (1993) 116

[15] M. Kuroda, F. M. Renard and D. Schildknecht, Phys. Lett. B198 (1987) 563

[16] A. de Rújula, M. B. Gavela, P. Hernández and E. Massó, Nucl. Phys. B384 (1992) 3

[17] P. Q. Hung and J. J. Sakurai, Nucl. Phys. B143 (1978) 81; Nucl. Phys. B148 (1979) 538 (Err.)

[18] M. Bilenky, J.-L. Kneur, F. M. Renard and D. Schildknecht, Bielefeld-Preprint BI-TP 92/25, to appear in Phys. Lett. B

[19] G. Gounaris, F. M. Renard and D. Schildknecht, Phys. Lett. B263 (1991) 291

[20] K. Gaemers and G. Gounaris, Z. Phys. C1 (1979) 259;

K. Hagiwara, R. D. Peccei, D. Zeppenfeld and K. Hisaka, Nucl. Phys. B282 (1987) 253

[21] C. N. Leung, S. T. Love and S. Rao, Z. Phys. C31 (1986) 433;

W. Buchmüller and D. Wyler, Nucl. Phys. B268 (1986) 621

[22] C. Bilchak, M. Kuroda and D. Schildknecht, Nucl. Phys. B299 (1988) 7

[23] H. Neufeld, J. D. Stroughair and D. Schildknecht, Phys. Lett. B198 (1987) 563

[24] K. Hagiwara, S. Ishihara, R. Szalapski and D. Zeppenfeld, Madison Preprint $\mathrm{MAD} / \mathrm{PH} / 737$ (1993)

[25] T. Appelquist and C. Bernard, Phys. Rev. D22 (1980) 200;

A. C. Longhitano, Nucl. Phys. B188 (1981) 118

[26] M. Chanowitz, M. Golden and H. Georgi, Phys. Rev. D36 (1987) 1490;

C. P. Burgess and D. London, McGill-Preprint McGill-92/04 (1992), hep-ph/9203215

[27] G. J. Gounaris and F. M. Renard, Montpellier-Preprint PM/92-31 (1992)

[28] C. Grosse-Knetter and R. Kögerler, Bielefeld-Preprint BI-TP 92/56 (1992), hep$\mathrm{ph} / 9212268$ 
[29] D. Barua and S. N. Gupta, Phys. Rev. D16 (1977) 413;

H. D. Politzer, Nucl. Phys. B172 (1980) 349;

G. Schäfer, Phys. Lett. A100 (1984) 128;

T. Damour and G. Schäfer, J. Math. Phys. 32 (1991) 127;

H. Georgi, Nucl. Phys. B361 (1991) 339;

H. Leutwyler, Bern-Preprint BUTP-91/26 (1991);

C. Arzt, Michigan-Preprint, UM-TH-92-28 (1992), hep-ph/9304230;

C. Grosse-Knetter, BI-TP 93/29 (1993), hep-ph/9306321

[30] C. Bilchak and D. Schildknecht, Z. Phys. C47 (1990) 595

[31] G. Altarelli, R. Barbieri and S. Jadach, Nucl. Phys. B369 (1992) 3

[32] J.-L. Kneur and D. Schildknecht, Nucl. Phys. B357 (1991) 357

[33] ALEPH, DELPHI, L3 and OPAL Collab., Phys. Lett. B276 (1992) 247

[34] CDF Collab., F. Abe et. al., Phys. Rev. Lett. 65 (1990) 2243;

UA2 Collab., J. Alitti et. al., Phys. Lett. B276 (1992) 354

[35] CDHS Collab., A. Blondel et. al., Z. Phys. C45 (1990) 361;

CHARM Collab., J. V. Allaby et. al., Z. Phys. C36 (1987) 611;

M. C. Noecker, B. P. Maesterson and C. E. Wieman, Phys. Rev. Lett. 61 (1988) 310

[36] E. Blucher et. al. (eds.), Report of the working group on high luminosities at LEP (CERN 91-02, Geneva, 1991)

[37] Talks by C. De Clerq and V. Innocente at the XXVIII Rencontres de Moriond on "Electroweak Interactions and Unified Theories", March 1993;

Talk by M. Pepe-Altarelli at the Rencontres de la Vallée d'Aoste, La Thuile, "Results and Perspectives in Particle Physics", March 1993 